\title{
DETERMINACIÓN DE LA RESISTENCIA NO DRENADA AL CORTE DE SUELOS ORGÁNICOS, MEDIANTE EL USO DEL CONO DE PENETRACIÓN ESTÁTICO Y EL DILATÓMETRO PLANO DE MARCHETTI
}

DETERMINATION OF THE UNDRAINED SHEAR STRENGTH OF ORGANIC SOILS USING THE CONE PENETRATION TEST AND MARCHETTI'S FLAT DILATOMETER TEST

JORGE ALBUJA ${ }^{1}$

Recibido: 18 de octubre de 2017 Aceptado: 22 de febrero de 2018

\footnotetext{
${ }^{1}$ Pontificia Universidad Católica del Ecuador, Facultad de Ingeniería, Quito, Ecuador (jdalbuja@puce.edu.ec).
} 



\section{DETERMINACIÓN DE LA RESISTENCIA NO DRENADA AL CORTE DE SUELOS ORGÁNICOS, MEDIANTE EL USO DEL CONO DE PENETRACIÓN ESTÁTICO Y EL DILATÓMETRO PLANO DE MARCHETTI}

\section{DETERMINATION OF THE UNDRAINED SHEAR STRENGTH OF ORGANIC SOILS USING THE CONE PENETRATION TEST AND MARCHETTI'S FLAT DILATOMETER TEST}

Jorge Albuja

Palabras clave: Suelos orgánicos; Turba; resistencia al corte; Cono de Penetración; Dilatómetro de Marchetti; Ensayos in situ;

Keywords: Organic Soils; Peat; Shear Resistance; Penetration cone test; Marchetti's Dilatometer; Field Tests.

\section{RESUMEN}

En los suelos orgánicos y turbas, métodos que en los suelos minerales, sin la determinación de la resistencia al corte no drenada es mediante los mismos embargo, no todas las metodologías son aplicables. Los suelos orgánicos presen- 
tan diferentes grados de descomposición, tipos de fibras, e incluso presencia de gas por los procesos de descomposición. Por esta razón, se ha realizado una comparación de la determinación de este parámetro en suelos orgánicos presentes en el sur de la Ciudad de Quito, mediante ensayos in situ como el Cono de Penetración Estático, el Dilatómetro de Marchetti y el Ensayo de Penetración Estándar, así como con ensayos de laboratorio. La investigación concluye con un cuadro comparativo de la resistencia al corte obtenida con cada ensayo.

\section{ABSTRACT}

In organic soils and peats, the determination of the undrained shear strength is done through the same methods used in mineral soils, however, not all the methodologies can be applied to this type of soils. Organic soils have different degrees of decomposition, different fibre types, and even the presence of gas by decomposition processes. For this reason, a comparison of the determination of this parameter in organic soils, which are present in the South of the City of Quito, has been carried out through in situ tests such as the Cone of Static Penetration, the Marchetti Dilatometer and the Standard Penetration Test, as well as with laboratory tests. The research concludes with a comparative table of the undrained shear strength obtained with each test.

\section{INTRODUCCIÓN}

La caracterización de suelos orgánicos, los cuales son depósitos de suelo con altos contenidos de agua y materia vegetal, han sido tema de discusión y preocupación para los ingenieros por décadas (A. O. Landva \& Pheeney, 1980; Mesri \& Ajlouni, 2007). En ingeniería civil, los suelos orgánicos y las turbas son suelos problemáticos, debido principalmente a los excesivos asentamientos que sufren las estructuras cimentados sobre ellos. Esto se produce cuando estos suelos son sometidos a esfuerzos de compresión, especialmente a largo plazo durante la consolidación secundaria (Meyer, Coufal, Kowalów, \& Szczygielski, 2011; Rahgozar \& Saberian, 2016), presentando deformaciones unitarias axiales que llegan al $50 \%$ debido a su enorme compresibilidad (Yang \& Liu, 2016). 
Su alta compresibilidad, baja resistencia al corte y compleja distribución de fibras ha causado que, terrenos con presencia de estos suelos, sean evitados en el pasado para su uso en construcción, al ser muy compleja su caracterización o muy altos los costos para cimentar sobre ellos (A. Landva \& La Rochelle, 1983)

En la actualidad, debido al creciente desarrollo urbano e industrial, no es posible evitar siempre este tipo de suelos, razón por la cual se aplica, donde sea posible, la remoción total o parcial de este suelo, el uso de pilotes o la aplicación de altos factores de seguridad, lo cual puede llevar a costos elevados o a impactos ambientales negativos (Kamao, 2016; Zwanenburg \& Jardine, 2015).

Debido a esta necesidad, para poder ejecutar obras de ingeniería civil sobre suelos orgánicos y turbas de forma sustentable, se requiere una adecuada caracterización mecánica de este tipo de materiales, tanto para estimar sus posibles asentamientos a corto y largo plazo, así como para estimar su resistencia al corte no drenada, la cual es necesaria para el diseño de cimentaciones durante y después de la construcción (Colleselli, Cortellazzo, \& Cola, 2000).

La caracterización de suelos orgánicos puede ser extremadamente compleja (Yang \& Liu, 2016), y en la bibliografía actual las investigaciones han sido direccionadas hacia la estimación de su compresibilidad, de la resistencia al corte no drenada, su estabilización y de su clasificación. (Landva and Pheeney, 1980; Burwash and Wiesner, 1984; Huat, 2014; Wong, 2014; Zhang and O'Kelly, 2014; Johari, Bakar and Aziz, 2015; O'Kelly, 2015; Hayashi, Nishimoto and Yamanashi, 2016).

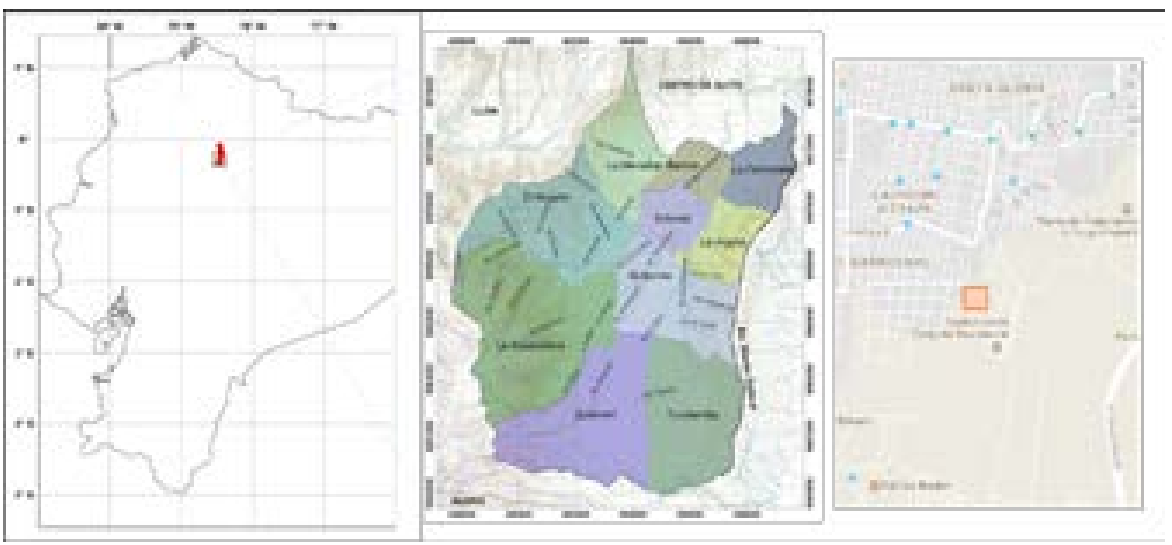

Figura n. ${ }^{\circ}$ 1. Ubicación del sitio de estudio (Avilés, 2013; Peñafiel, 2008; modificado) 
Considerando que la resistencia al corte no drenada es de vital importancia para el diseño de cimentaciones de obras civiles sobre suelos orgánicos, sobre todo cuando ésta es muy baja (Colleselli et al., 2000), se estimará dicha resistencia, mediante el uso de metodologías in situ relativamente nuevas, como son el caso del Cono de Penetración Estático y del Dilatómetro de Marchetti, los cuales ya han sido utilizados para la caracterización de suelos orgánicos (A. Elsayed, 2006; Marchetti, 1980; Mayne, Coop, Springman, Huang, \& Zornberg, 2009; Zawrzykraj, Rydelek, \& Bąkowska, 2017), así como del ensayo de Penetración Estándar (Standard Penetration Test - SPT), el cual es el método de ensayo de mayor uso, extensión y difusión, utilizado tanto en Quito como en todo el Ecuador, para la exploración y caracterización geotécnica (Ludeña, 2007).

Los resultados de estos ensayos in situ serán comparados con ensayos de laboratorio, así como con datos bibliográficos, con el fin de determinar qué tipo de metodología es aplicable y adecuada para la obtención de la resistencia al corte no drenada de suelos orgánicos.

\section{CARACTERÍSTICAS DEL SITIO DE ANÁLISIS}

Los suelos orgánicos se forman por la deposición de remanentes de plantas, animales y organismos, los cuales se acumulan en zonas con poco drenaje, lugares con excesiva Iluvia, como las zonas tropicales, o sitios donde las bajas temperaturas no permiten una descomposición aeróbica de estos remanentes (Huat, 2014; Yamaguchi, Kawano, Teranishi, Matsui, \& Miura, 1988) Este fenómeno provoca que estos fragmentos se acumulen bajo el agua y se fosilicen (Dhowian \& Edil, 1980; Edil, Fox, \& Lan, 1991; Mesri \& Ajlouni, 2007; Radforth \& Radforth, 1965; Soper \& Osbon, 1922; Yang \& Liu, 2016) 


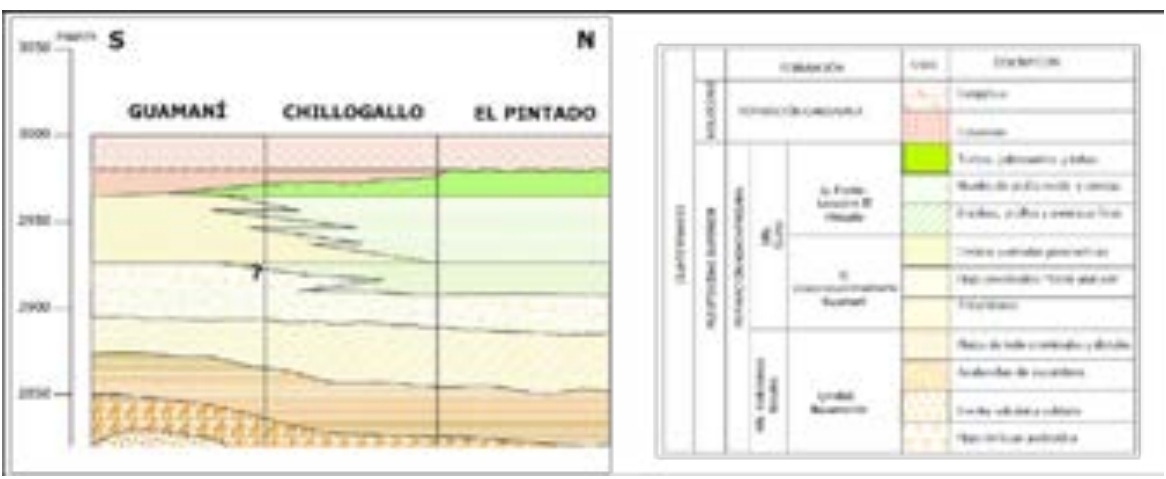

Figura n. ${ }^{2}$ 2. Litología subcuenca sur - Quito (Avilés, 2013; Peñafiel, 2008; modificado)

Los sitios con presencia de suelos orgánicos y turbas pueden ser encontrados en prácticamente todos los países del mundo (Parish et al., 2008), su presencia es registrada con mayor detalle en países desarrollados como Escocia, con sobre el $60 \%$ de presencia con respecto a su área total (Joint Nature Conservation Committee, 2011), Finlandia con el $30 \%$, Canadá con $18 \%$, Irlanda con $17 \%$, Rusia y Canadá con el $10 \%$, y en Estados Unidos en 42 estados (A. A. Elsayed, 2003; Mesri \& Ajlouni, 2007; Parish et al., 2008) En el caso de países en vías de desarrollo, como Colombia, Ecuador y Perú, es únicamente conocido de forma aproximada, con estimaciones del 0.5 al $5 \%$ con respecto a su área total (A. A. Elsayed, 2003).

El presente estudio se centra en el sector sur de la ciudad de Quito, en la zona denominada "El Garrochal", ubi- cada a $2.5 \mathrm{~km}$ al norte de la Quebrada "Saguanchi" (Fig. 1). La ciudad de Quito se encuentra en una depresión topográfica, formando una cuenca que lleva su mismo nombre, en una dirección N-S. Esta cuenca constituida por la actividad del sistema de fallas inversas de Quito, lo cual ha generado una serie de lomas alargadas en los bordes de la ciudad (Villagómez, 2014). Esta cuenca tiene aproximadamente $30 \mathrm{~km}$ de largo y hasta $5 \mathrm{~km}$ de ancho, la cual a su vez está divida en 2 subcuencas, la primera denominada subcuenca centro - norte y la segunda denominada subcuenca sur, divididas por el río Machángara, y característicamente por el domo "El Panecillo" (Alvarado, 1996; Peñafiel, 2008; Villagómez, 2014)

La subcuenca sur tiene un área aproximada de 127.7 kilómetros cuadrados, dentro de los cuales 53.7 kilómetros 
conforman el valle del sur de Quito, y el cual es conocido como "Turubamba" sitio donde se encuentra la zona de "El Garrochal". La subcuenca sur está conformada por un sistema de quebradas que drenan hacia el Río "Machángara" al norte, y a la quebrada "Saguanchi" al sur, con una altura variable entre 3080 y 2800 m.s.n.m. (Peñafiel, 2008) Es en esta zona denominada "Turubamba" donde previamente existió una laguna, la cual durante su drenaje hacia el río Machángara - al no drenarse por completo - dejó un nivel freático alto, con vestigios de material orgánico, formando un terreno pantanoso razón por la cual fue llamado por los incas "Turubamba", lo cual se traduce en "Llano de lodo" o "Tierra de Pantanos" (Cuvi, 2017; Santander, 2013). Actualmente esta zona es parte de la unidad "Fluvio Lacustre El Pintado", formada por un conjunto de brechas volcánicas intercaladas por areniscas y capas de arcillas cuya base está sobre la unidad Volcanosedimentaria Guamaní, las cuales se intersecan y superponen desde el norte hacia el sur de la subcuenca sur. Sobre este conjunto de brechas se encuentran estratos de arcilla y arena de color verde, llegando a un espesor de 10 metros.

Finalmente se encuentra una serie de turbas, paleosuelos y tobas que alcanzan a un espesor de 20 metros (Peñafiel, 2008). Es justamente sobre esta última serie de turbas donde se han construido en las últimas décadas complejos habitacionales y de servicios públicos, los cuales han sufrido constantes problemas por asentamientos, lo cual ha sido causa de análisis por el sector público y privado, y es donde se ejecutará la presente investigación.

\section{MATERIALES Y METODOLOGÍA}

Los equipos utilizados incluyen a un Cono de Penetración Estático (Cone Penetration Test - CPT), conformado con un cono mecánico Begemann, un Dilatómetro Plano de Marchetti (Marchetti's Dilatometer Test - DMT) y el equipo para ejecutar el Ensayo de Penetración Estándar (Standard Penetration Test - SPT). Una unidad hidráulica Tecnotest con una máxima fuerza de 200 kN fue utilizada para hincar a ambos grupos de medición en el suelo. Los ensayos fueron realizados en una locación del sector denominado "El Garrochal", en el Sur de Quito (Fig. 1), con 3 ensayos CPT, 1 ensayo DMT y 1 ensayo SPT, con un total de 5 ensayos in situ ejecutados. Los sitios de cada ensayo fueron separados entre 1.5 a 2 metros; los resultados que se presentan son el promedio de los valores obtenidos en 
cada uno de los ensayos. En las perforaciones SPT se recolectaron muestras de suelo orgánico para determinación de porcentajes de materia orgánica, humedad y clasificación.

Los siguientes parámetros fueron registrados durante la ejecución de los ensayos CPT y DMT:

- CPT: Medición de la resistencia a la punta del cono qc resistencia a la fricción de la camisa $f_{s}$ con datos tomados cada $20 \mathrm{~cm}$ para ambas lecturas.

- DMT: Medición de las presiones $p_{0}$ y $p_{1}$ cada $20 \mathrm{~cm}$ en los perfiles verticales.

Los procedimientos para realizar los mencionados ensayos, así como la geometría de la punta del cono, de la camisa de fricción, y del dilatómetro plano de Marchetti, fueron ejecutados a base de las normas ASTM respectivas (ASTM D3441 - 16, 2014; ASTM D6635 - 15, 2016).

A base de la información registrada en el campo, se calcularon los siguientes valores para el CPT:

$$
q_{c}=1000 * Q_{c} / A_{c}
$$

Donde $q_{c}$ es la resistencia del cono en $\mathrm{MPa}, \mathrm{Q}_{c}$ es la fuerza de empuje medida en la punta del cono $(\mathrm{kN})$ y $A_{c}$ es el área de la base del cono, la cual equivale a $1000 \mathrm{~mm}^{2}$. Los resultados pueden ser observados en los 3 primeros esquemas del gráfico n. ${ }^{3} 3$.

$$
\begin{gathered}
f_{s}=1000 * F_{s} / A_{s} \\
F_{s}=Q_{f} / Q_{c}
\end{gathered}
$$

Donde $f_{s}$ es la resistencia de la camisa de fricción (MPa), $F_{s}$ es la fuerza de empuje medida en la camisa de fricción $(\mathrm{kN}), Q_{f}$ es la fuerza de empuje medida en la punta del cono más la camisa de fricción, y $A_{s}$ es el área de la camisa de fricción, equivalente a $15000 \mathrm{~mm}^{2}$.

$$
R_{f}=100 * f_{s} / q_{c}
$$

Donde $R_{f}$ es la relación de fricción, presentada como porcentaje.

Para el caso del DMT, las lecturas A y $B$ son corregidas por la rigidez de la membrana para predeterminar las presiones $p o$ y $p 1$, las cuales son aplicadas al suelo al inicio y al final de la expansión. Las siguientes expresiones son usadas:

$$
\begin{aligned}
& p o=A+\Delta A \\
& p 1=B-\Delta B
\end{aligned}
$$

Donde $\triangle \mathrm{A}$ es la presión externa que debe ser aplicada a la membrana al aire libre para mantenerla en contacto 
con su base y $\triangle \mathrm{B}$ es la presión interna la cual infla el centro de la membrana 1.0 $\mathrm{mm}$ desde su base. Los valores $\triangle \mathrm{A}$ y $\triangle \mathrm{B}$ son medidos mediante un procedimiento en campo, el cual es aplicar un vacío y una presión en la membrana respecti- vamente y leer los valores para los cuales la membrana se desinfla o infla $1.0 \mathrm{~mm}$ (ASTM D6635 - 15, 2016)

$\triangle A=0.15 \pm 0.05 \mathrm{~kg} / \mathrm{cm}^{2}$ $\Delta B=0.5 \pm 0.2 \mathrm{~kg} / \mathrm{cm}^{2}$

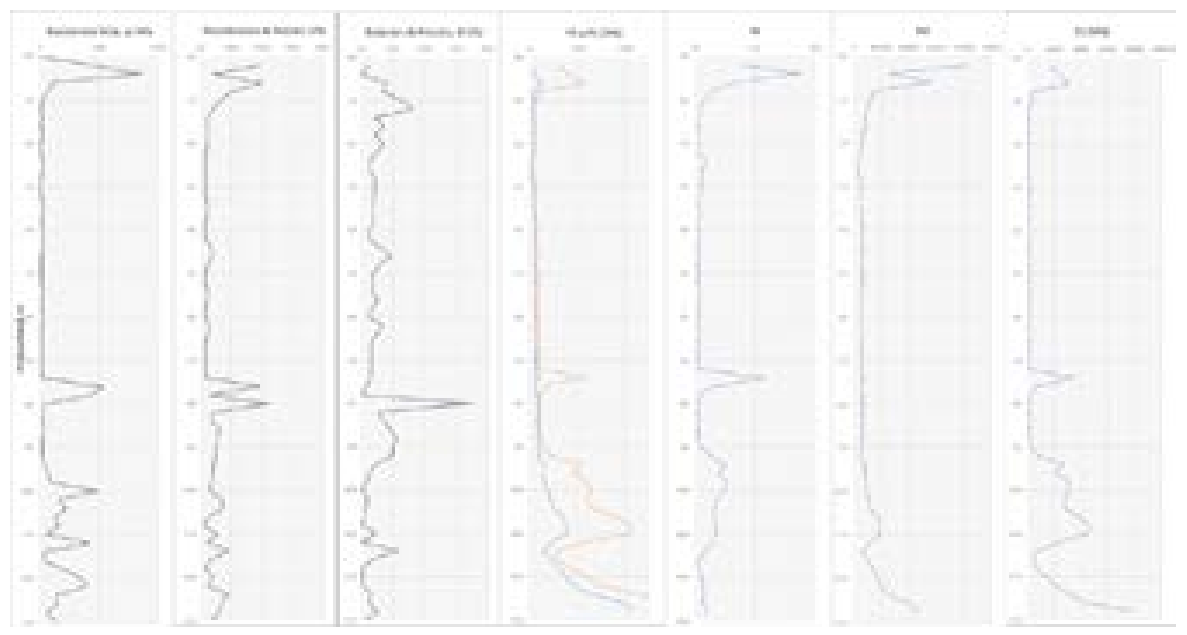

Figura n. ${ }^{\circ}$. Interpretación de resultados del CPT y DMT, hasta los 13 metros de profundidad

Los resultados del DMT son presentados como diagramas de po, p1 versus la profundidad, y como diagramas de los parámetros calculados ID, KD, y ED como se define a continuación:

Índice de Material:

$$
I_{D}=\frac{\Delta \mathrm{p}}{p_{o}-u_{o}}
$$

Índice de Esfuerzo Horizontal:

$$
K_{D}=\frac{p_{o}-u_{o}}{\sigma_{v}}
$$

Módulo del Dilatómetro:

$$
E_{D}=38.2 * \Delta p
$$

Donde $u_{o}$ y $\sigma_{v}$ son la presión de poros del agua y esfuerzo efectivo vertical respectivamente, y deben ser conocidos, al menos de forma aproximada, 
previo a la inserción de la cuchilla. Los resultados pueden ser observados en los 4 últimos esquemas del gráfico n. ${ }^{\circ} 3$.

En el ensayo SPT, se determinó el número de golpes " $\mathrm{N}$ " necesarios para introducir el muestreador denominado cuchara partida a una profundidad de $30 \mathrm{~cm}$ mediante un martinete de $64 \mathrm{~kg}$ de masa que cae desde una altura de 76 centímetros, información que se puede observar en el perfil estratigráfico de la figura n.o 3 (ASTM D1586-11, 2008). De los primeros 7 metros, donde se presen- ció la mayor cantidad de suelo orgánico y hasta donde se recuperaron muestras alteradas a partir del ensayo SPT, se lo caracterizó mediante ensayos de cantidad de materia orgánica, contenido de humedad y límites de Atterberg.

Los parámetros estadísticos obtenidos de ambos ensayos se presentan en las figuras 3 y 4, correspondientes a la resistencia a la punta del cono $q_{c}$ y al índice del material del DMT $I_{D}$. Para la determinación de la resistencia no drenada al corte, se utilizaron las siguientes expresiones:

- $\quad C u_{C P T}=\left(q_{c}-\sigma_{v o}\right) / N_{K T}[M P a]$ (Lunne, Robertson, \& Powell, 1997)

- $C u_{D M T}=0,22 * \sigma_{v o} *(0,5 * K d)^{1,25}[M P a]$ (Marchetti, 1980)

- $C u_{L A B}=\frac{q u}{2}[M P a]$

Donde: $\sigma_{v o}$ es presión de sobrecarga en la profundidad medida [MPa], $N_{K T}$ es un coeficiente empírico según el tipo de suelo, $N_{60}$ es el $N$ con un $60 \%$ de la energía teórica transmitida, y $q_{u}$ es la resistencia más alta obtenida de los ensayos de compresión simple, aplicando el modelo de Tresca para la obtención de $\mathrm{Cu}$.
Para el caso del ensayo SPT, se aplicaron las correlaciones existentes para suelos blandos, al no haber correlaciones directas para turbas o suelos orgánicos en la bibliografía existente. A continuación se presentan las ecuaciones y tablas utilizadas para las correlaciones: 


\begin{tabular}{|l|l|l|}
\hline Consistencia & N SPT & $\mathbf{q}_{\mathbf{u}}(\mathbf{k P a})$ \\
\hline Muy blanda & $<2$ & $<25$ \\
\hline Blanda & $2-4$ & $25-50$ \\
\hline Media & $4-8$ & $50-100$ \\
\hline Rígida & $8-15$ & $100-200$ \\
\hline Muy Rígida & $15-30$ & $200-400$ \\
\hline Dura & $>30$ & $>400$ \\
\hline
\end{tabular}

Tabla n. ${ }^{1}$ 1. Correlación entre N SPT y qu (kPa) (Terzaghi, Peck, \& Mesri, 1996)

\begin{tabular}{|c|c|c|}
\hline Autores & Tipo de Suelo & $\mathrm{Su}(\mathrm{kPa})$ \\
\hline \multirow{2}{*}{ Sanglerat (1972) } & Arcilla & $12.5 \mathrm{~N}$ \\
\hline & Arcilla Limosa & $10 \mathrm{~N}$ \\
\hline Terzaghi \& Peck (1967) & Suelo de grano fino & $6.25 \mathrm{~N}$ \\
\hline Hara et al. (1974) & Suelo de grano fino & $29 \mathrm{~N}_{60} .072$ \\
\hline \multirow{3}{*}{ Sowers (1979) } & Suelo altamente plástico & $12.5 \mathrm{~N}$ \\
\hline & Suelo de mediana plasticidad & $7.5 \mathrm{~N}$ \\
\hline & Suelo de baja plasticidad & $3.75 \mathrm{~N}$ \\
\hline Nixon (1982) & Arcilla & $12 \mathrm{~N}$ \\
\hline \multirow{3}{*}{ Sivrikaya \& Toğrol (2002) } & Suelo altamente plástico & $6.82 \mathrm{~N}_{60}$ \\
\hline & Suelo de baja plasticidad & $4.93 \mathrm{~N}_{60}$ \\
\hline & Suelo de grano fino & $6.18 \mathrm{~N}_{60}$ \\
\hline \multirow{3}{*}{ Stroud (1974) } & $\mathrm{Pl}<20$ & $(6-7) N$ \\
\hline & $20<\mathrm{PI}<30$ & $(4-5) \mathrm{N}$ \\
\hline & $\mathrm{PI}>30$ & $4.2 \mathrm{~N}$ \\
\hline Décourt (1990) & Arcilla & $15 \mathrm{~N}_{60}$ \\
\hline Ajayi \& Balogun (1988) & Suelo de grano fino & $1.39 N+74.2$ \\
\hline Hettiarachchi \& Brown (2009) & Suelo de grano fino & $4.1 \mathrm{~N}_{60}$ \\
\hline Sirvikaya (2009) & Ensayo de compresión simple sin confinamiento & $S u=2.41 \mathrm{~N}-0.82 \mathrm{wn}+0.14 \mathrm{LL}+1.44 \mathrm{PI}$ \\
\hline
\end{tabular}

Tabla n. ${ }^{\circ}$ 2. Correlaciones entre N SPT y Su (kPa) (Nassaji, 2011) 
Para la determinación de Su mediante correlaciones con el $\mathrm{N}$ del SPT, se aplicaron todas las correlaciones, y de ser el caso, se buscó una posible correlación entre los resultados obtenidos mediante laboratorio (ensayos de compresión sin confinar), cuyos resultados se presentan en la siguiente sección.

\section{RESULTADOS}

Los suelos estudiados presen- el procedimiento recomendado por la tan un contenido orgánico promedio norma ASTM D2974-14 "Tests for deterde $26.89 \%$, los cuales de acuerdo con el mination of moisture, ash and organic sistema de clasificación de Landva (A. O. matter of peats", donde se mantuvo una Landva, Korpijaakko, \& Pheeney, 1983), temperatura de $110^{\circ} \mathrm{C}$ hasta que no exisse clasifican como suelos orgánicos, y un ta cambio de masa, lo cual tomó 24 hocontenido de humedad de $269.7 \%$ en los ras, para posteriormente aplicar $440{ }^{\circ} \mathrm{C}$ primeros 7 metros prospectados. Ambos hasta que no exista cambio de masa, lo contenidos fueron obtenidos mediante cual tomó 7 horas. 

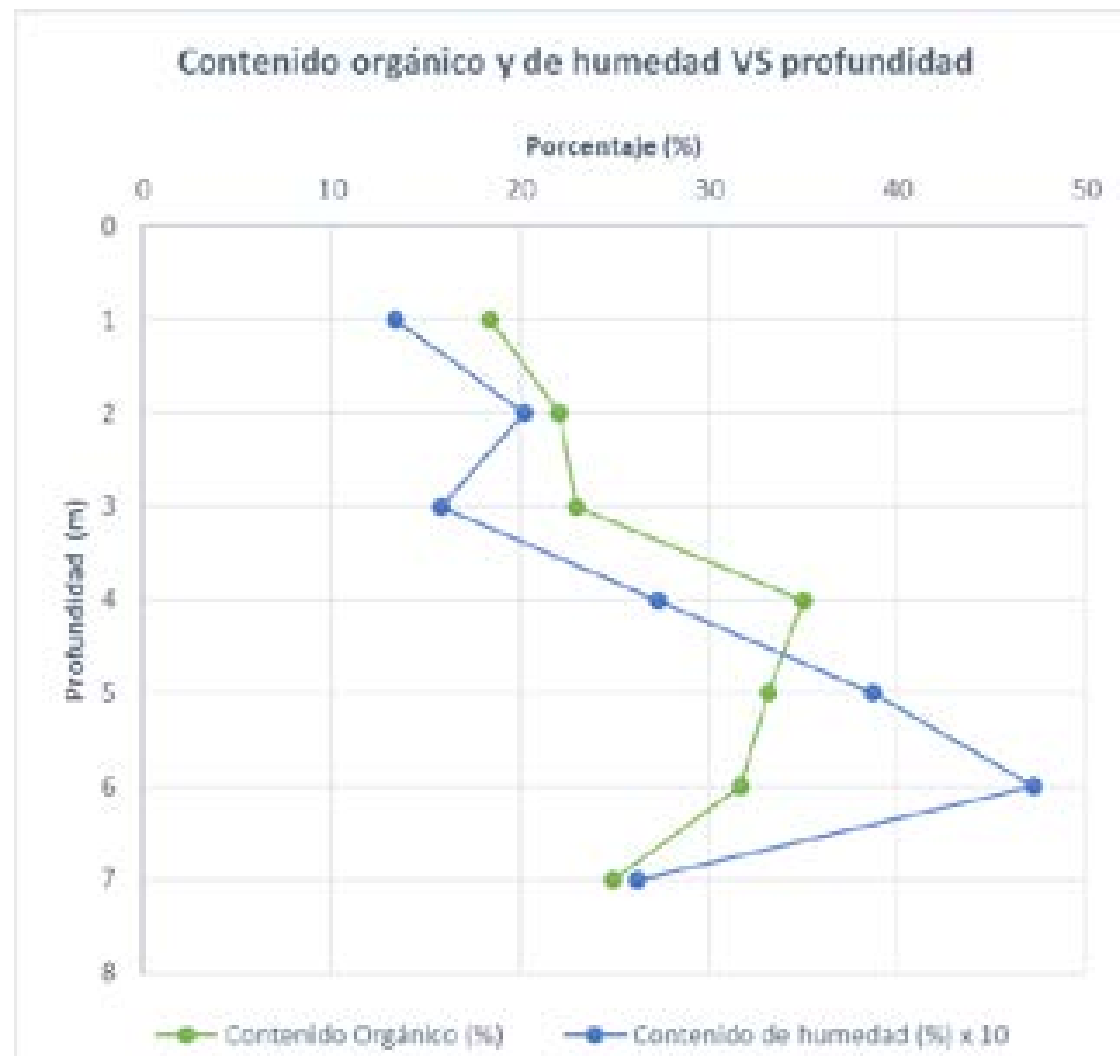

Figura n. ${ }^{\circ}$. Contenido orgánico y de humedad (\%) versus profundidad

Previo a la aplicación de esta metodología, se estudiaron otros métodos para la determinación de la materia orgánica (Jarrett, 1983; A. O. Landva et al., 1983; Skempton \& Petley, 1970), la cual tiene una cercanía a la metodología presentada por Landva et. al, 2013, donde se propone aplicar $440{ }^{\circ} \mathrm{C}$ por cinco horas para determinar el contenido de materia orgánica.

Los resultados se presentan en el gráfico n.o 4., donde el contenido de humedad fue multiplicado por diez para que pueda ser comparado con el contenido de humedad. Sin tomar en cuenta el quinto y sexto metro de profundidad, 
debido a que existían presencia de arenas que redujeron la cantidad de materia orgánica, se puede observar que ambos contenidos guardan una cercana relación en su variación, con diferencias en promedio del $4.1 \%$.

Similares relaciones ya han sido investigadas previamente obteniendo un comportamiento similar (Haan \& Kruse, 2007), lo cual puede ser de gran utilidad cuando no existen medidas de esfuerzos en campo o en laboratorio (Boylan, Noel; Long, 2012) así como lo complicado que puede ser una estratigrafía, donde al existir muchas variaciones en los tipos de material, como son el caso del 5 to y 6 to metro de profundidad, la relación entre ambos contenidos puede ser de poca utilidad (Nichol, 1998a).

Sobre estas muestras se determinaron los límites de Atterberg (Fig. 5), los cuales son indicadores de los rangos de contenido de humedad en el cual la consistencia de un suelo pasa de un estado líquido, a plástico y finalmente a sólido (Terzaghi et al., 1996).

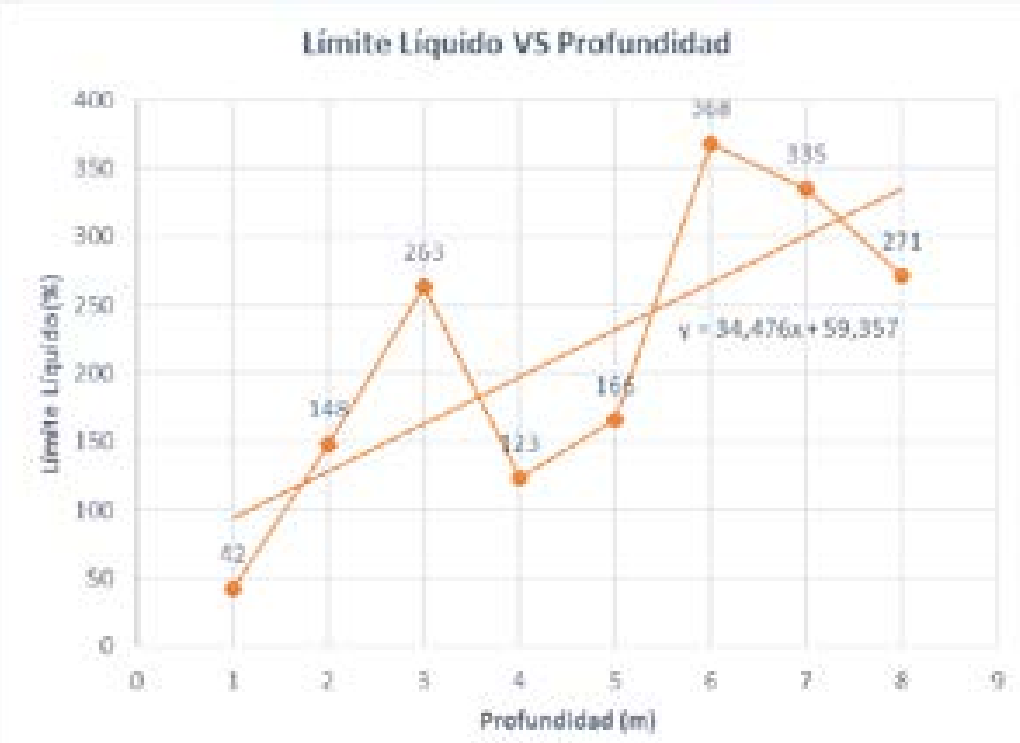

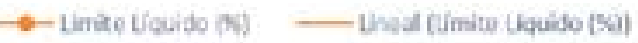

Figura n. ${ }^{0}$. Límite Líquido (\%) versus profundidad 
En el caso de los suelos orgánicos y las turbas, su determinación ha sido estudiada reportada como complicada, reportando valores erráticos y de complicada interpretación (Ajlouni, 2000; Skempton \& Petley, 1970; Yamaguchi, Ohira, Kogure, \& Mori, 1985), y en otros casos, se recomienda no utilizar los límites de Atterberg para este tipo de suelos (O'Kelly, 2015). Sin embargo a lo antes mencionado, se realizaron los ensayos de límites, y también se calculó la estimación de contenido orgánico en base al límite líquido, mediante la ecuación de Skempton $(\mathrm{LL}-50)=5^{*} \mathrm{~N}$ donde $\mathrm{N}=$ Contenido Orgánico (Skempton \& Petley, 1970), cuyos resultados se pueden observar en la figura n. 6. y los cuales no guardan una correlación, compartiendo una aproximación únicamente de los valores de materia orgánica en el tercer y cuarto metro de profundidad.

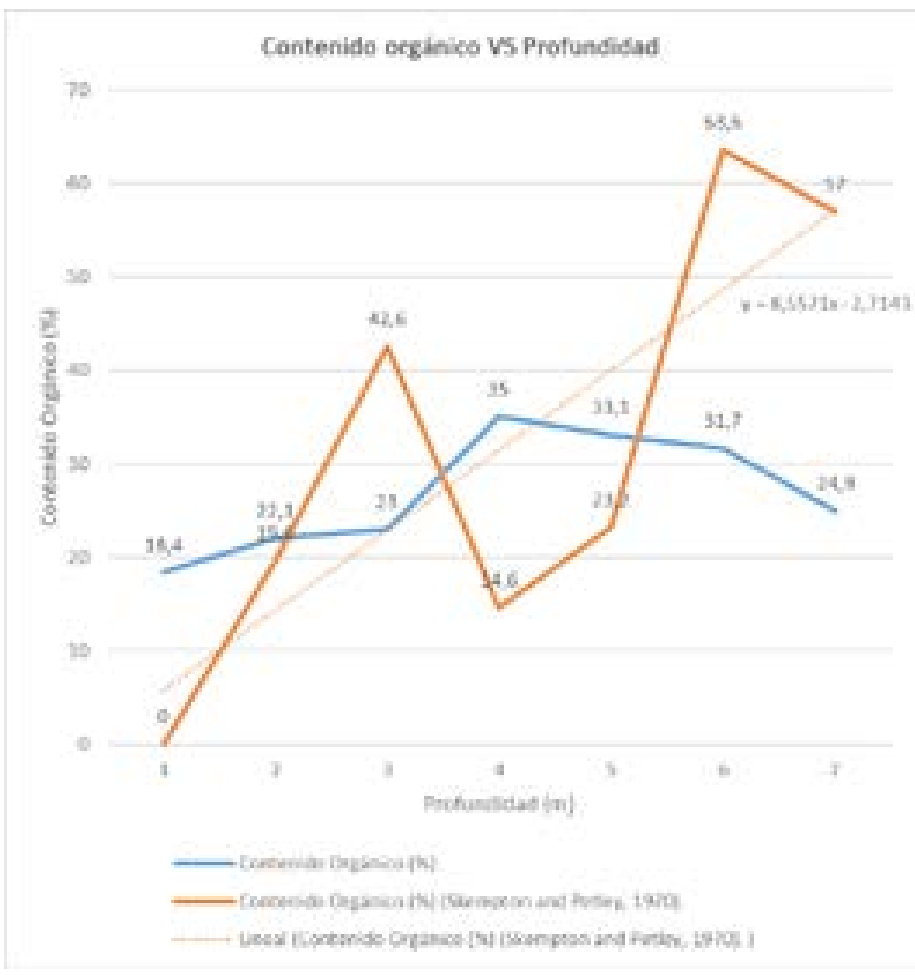

Figura n. ${ }^{\circ} 6$. Contenido Orgánico (\%) versus profundidad 
Las lecturas obtenidas de los en- chetti \& Crapps, 1981; Robertson, Camsayos CPT y DMT fueron graficadas en los panella, Gillespie, \& Greig, 1986), lo cual nomogramas de predicción de tipo de puede ser observado en las figuras 9 y 10 suelo que usualmente se aplican (Mar- respectivamente.

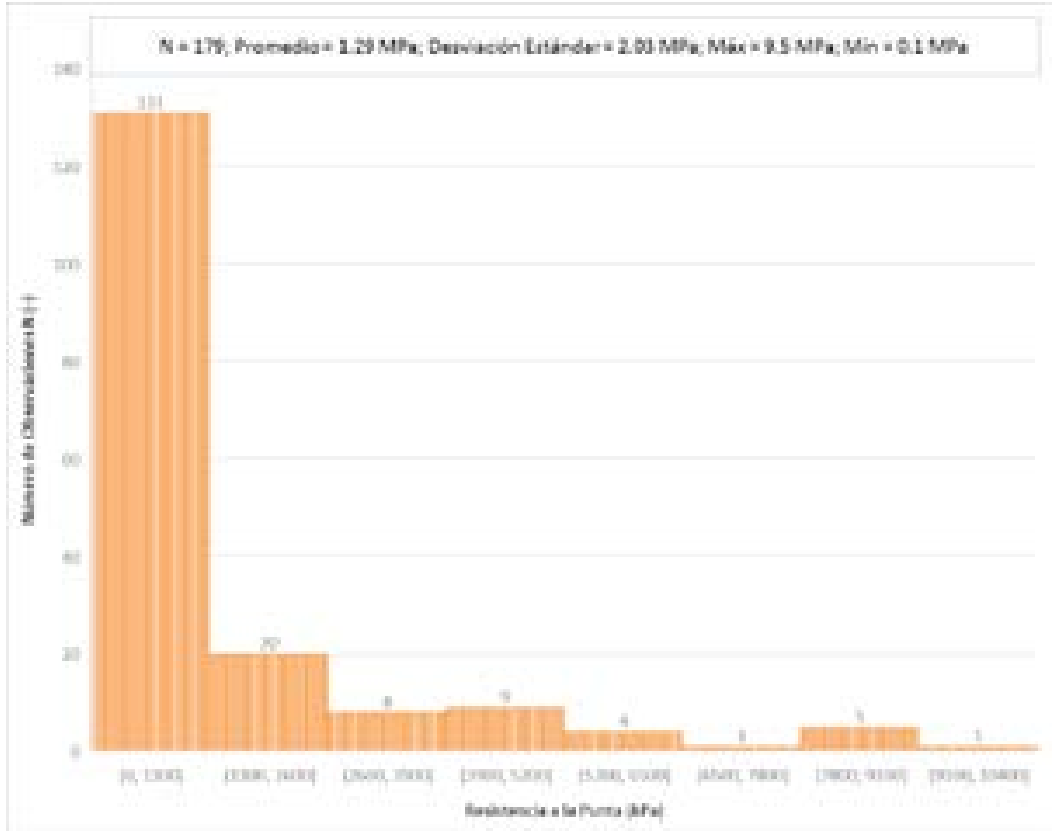

Figura n. ${ }^{\circ}$ 7. Datos estadísticos ensayo (PT - Resistencia a la Punta (kPa)

A partir de los resultados obte- Robertson y Campanella (Robertson et nidos del ensayo CPT presentados en el gráfico n. 3 , se obtuvieron las estadísticas presentadas en el gráfico n. 8 , donde la resistencia a la punta menor a 1300 kPa representa el $73 \%$ del total de los datos. A partir de los mismos resultados, se aplicó el nomograma propuesto por al., 1986), con modificaciones y con una mayor extensión en el eje horizontal, debido a que se tienen valores de Relación de Fricción que llegan a $150 \%$. La aplicación de este nomograma dio resultados de tipos de suelo diferentes a los actualmente encontrados, presentando 
más del $70 \%$ de los suelos como suelos minerales, por lo que, en el caso de los suelos orgánicos, el uso del nomograma de Robertson puede dar resultados de caracterización erróneos en cerca del
$70 \%$, y los que son caracterizados correctamente, con valores de relación de fricción FR muy por encima del $8 \%$ que delimita el nomograma original.

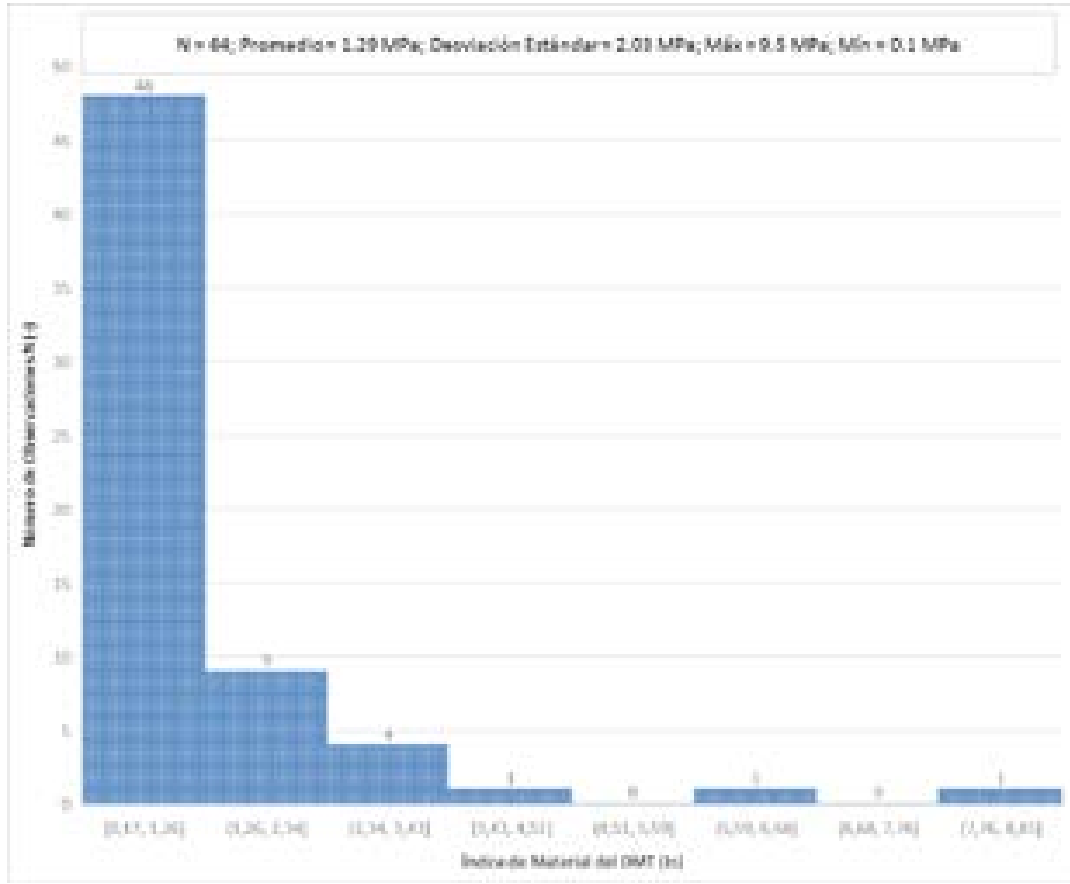

Figura n. ${ }^{8}$ 8. Datos estadísticos ensayo DMT

De los resultados obtenidos del ensayo DMT del gráfico n. ${ }^{3} 3$, se obtuvieron las estadísticas presentadas en el gráfico n. 8 , donde valores de índice de material menores a 1.26 representan el $75 \%$ del total. A partir de los mismos resultados, se aplicó el nomograma pro- puesto por Marchetti y Crapps (Marchetti \& Crapps, 1981) y presentado en la norma ASTM (ASTM D6635 - 15, 2016), lo cual se puede observar en la figura n. 10 , donde se obtuvo que el $18 \%$ de los puntos fueron clasificados como suelos orgánicos o turbas, el $45 \%$ presen- 
taron puntos cercanos a los clasificados ficados como arcillas, y el $55 \%$ restante como turbas, sin embargo, fueron clasi- como suelos minerales en general.

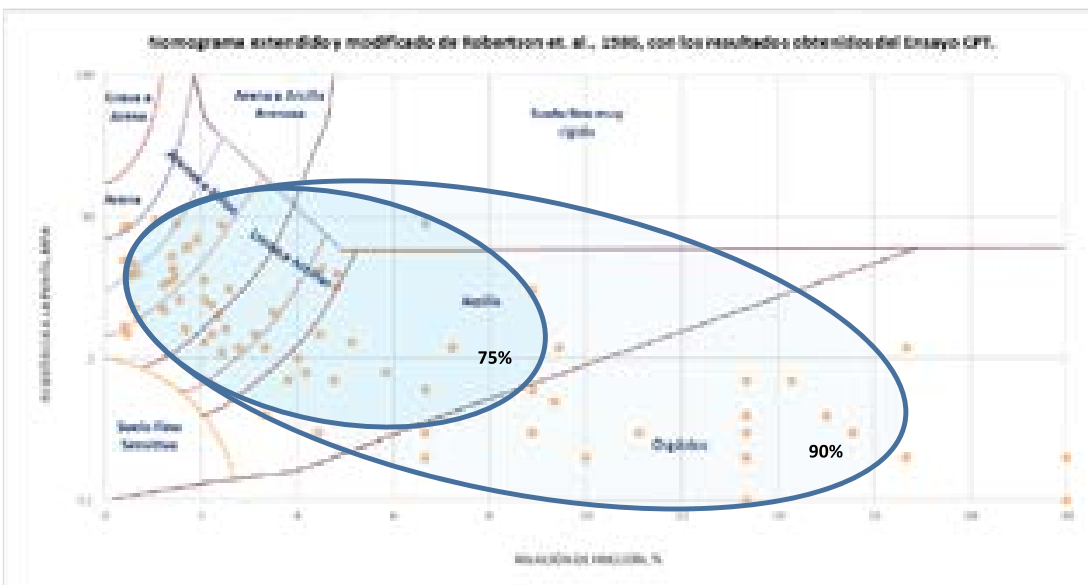

Figura n. ${ }^{9}$ 9. Interpretación de resultados DMT en el nomograma de predicción de tipo de suelo (Robertson, Campanella, Gillespie, \& Greig, 1986), modificado.

Considerando la obtención de datos tanto del ensayo CPT y DMT, se buscó una correlación entre la resistencia del cono del CPT, $q_{c}$, con el módulo edométrico $M_{D M T}$, con base en la fórmula general que permite correlacionar ambas $M_{D M T}=\alpha^{*} q_{c}$. En la figura n. ${ }^{\circ} 11$ se puede observar una línea de tenden- cia linear donde se obtiene un valor de $\alpha=8.455$, cercano a $\alpha=8.5$, el cual se obtuvo con datos únicamente hasta los 12.8 metros de profundidad, al existir una variabilidad muy alta a mayor profundidad, por lo cual se propone un valor de $\alpha=8.5$ para los presentes suelos estudiados. 


\section{SOIL DESCRIPTION and ESTIMATED $\gamma / \gamma_{\mathrm{w}}$}

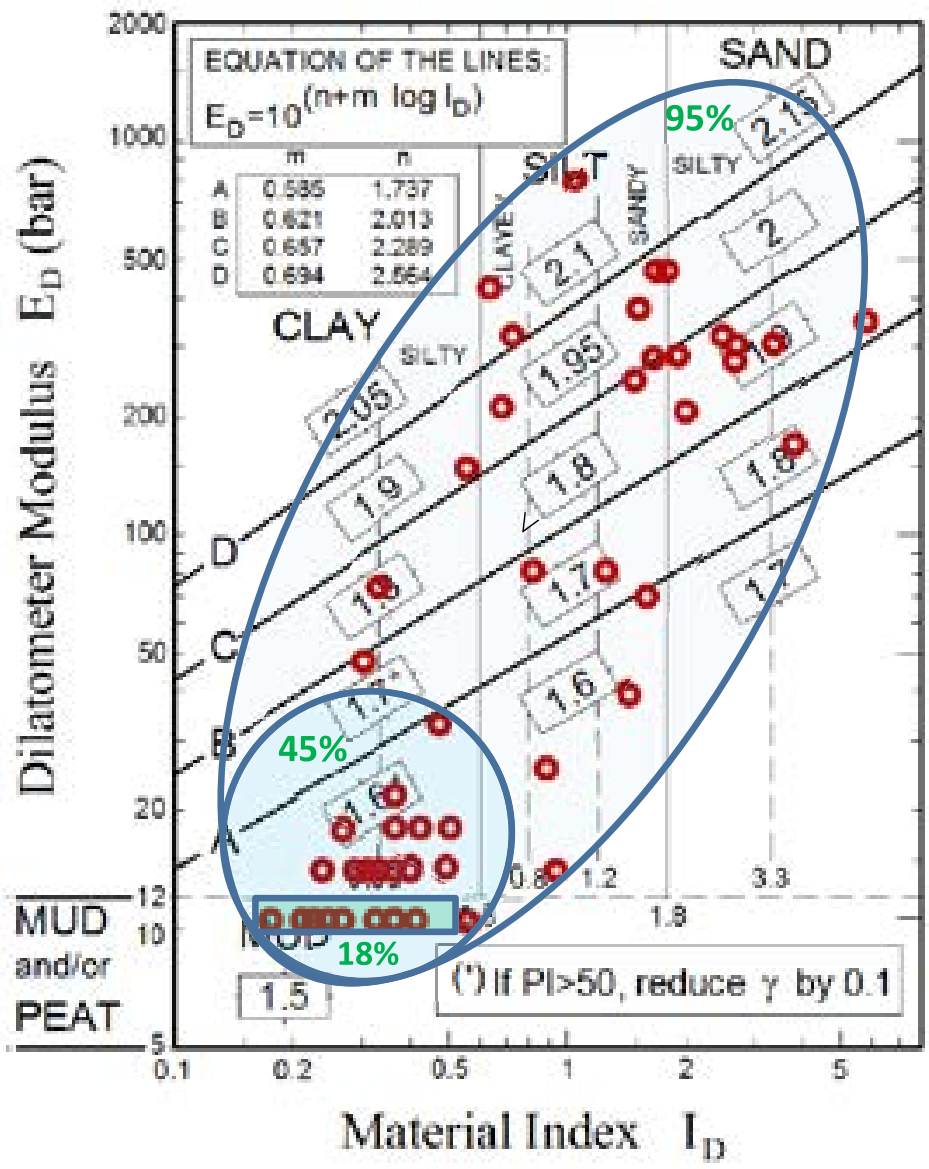

Figura n. ${ }^{\circ}$ 10. Nomograma propuesto por Marchetti y Crapps para identificación de tipos de suelo (ASTM D6635 $-15,2016$; Marchetti \& (rapps, 1981) 
Finalmente, se aplicaron las ecuaciones para la obtención de la resistencia al corte no drenada Cu indicadas en la Metodología, de las cuales se obtuvo como resultado el cuadro comparativo indicado en la figura n. ${ }^{\circ} 12$, con rangos de Cu hasta de $300 \mathrm{kPa}$. Una clara simili- tud de valores se observa entre los resultados del DMT, CPT y ensayos de Laboratorio, cuyos rangos de Cu no superan los $40 \mathrm{kPa}$ y se encuentran sombreados del primero al noveno metro de prospección, que es donde se presentó la concentración de suelo orgánico.

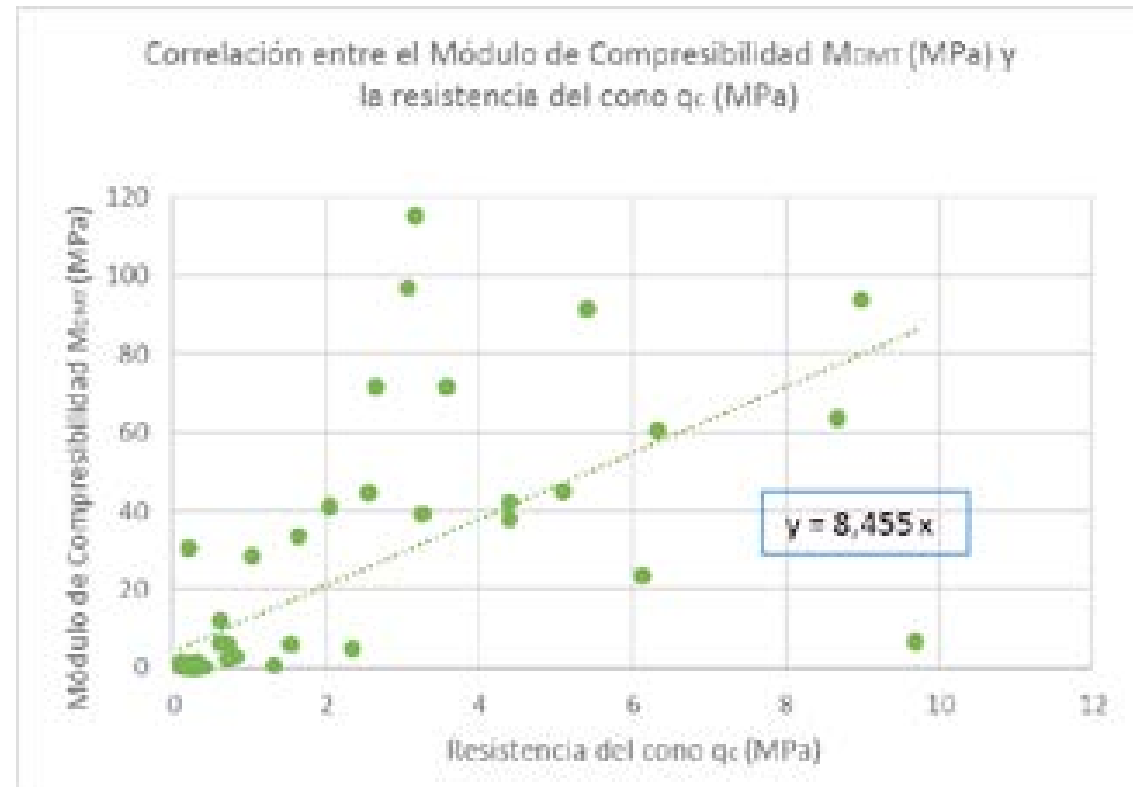

Figura n. ${ }^{0}$ 11. Correlación entre $q_{c} \mathrm{y} M_{D M T}$.

En la figura 12 se observa una clara sobre estimación del valor de $\mathrm{Cu}$ estimados mediante el ensayo SPT en toda la profundidad prospectada, con un claro ejemplo en el tercer metro de prospección, donde los rangos de los ensayos de laboratorio van de 7 a $25 \mathrm{kPa}$, del ensayo CPT de 9 a 26 kPa, y del ensayo DMT de 5 a $11 \mathrm{kPa}$, mientras que con el ensayo SPT se obtiene un valor de 47.8 $\mathrm{kPa}$, lo cual representa en promedio un incremento del $300 \%$ del valor estimado 
de Cu con respecto a los otros ensayos. En el sexto y séptimo metro este incremento en la estimación del Cu llega a valores superiores al $2500 \%$ con respecto a los valores estimados con el CPT y el DMT.
Los valores de resistencia al corte no drenada (Su) en laboratorio fueron obtenidos mediante ensayos de compresión simple no confinados, donde se aplicó el criterio de Tresca $C u_{L A B}=\frac{q u}{2}$ [MPa]. Los resultados de laboratorio son los siguientes:

- Muestra P1 de 2,50 a 3,00 m (2,50 a 2,60) de profundidad.

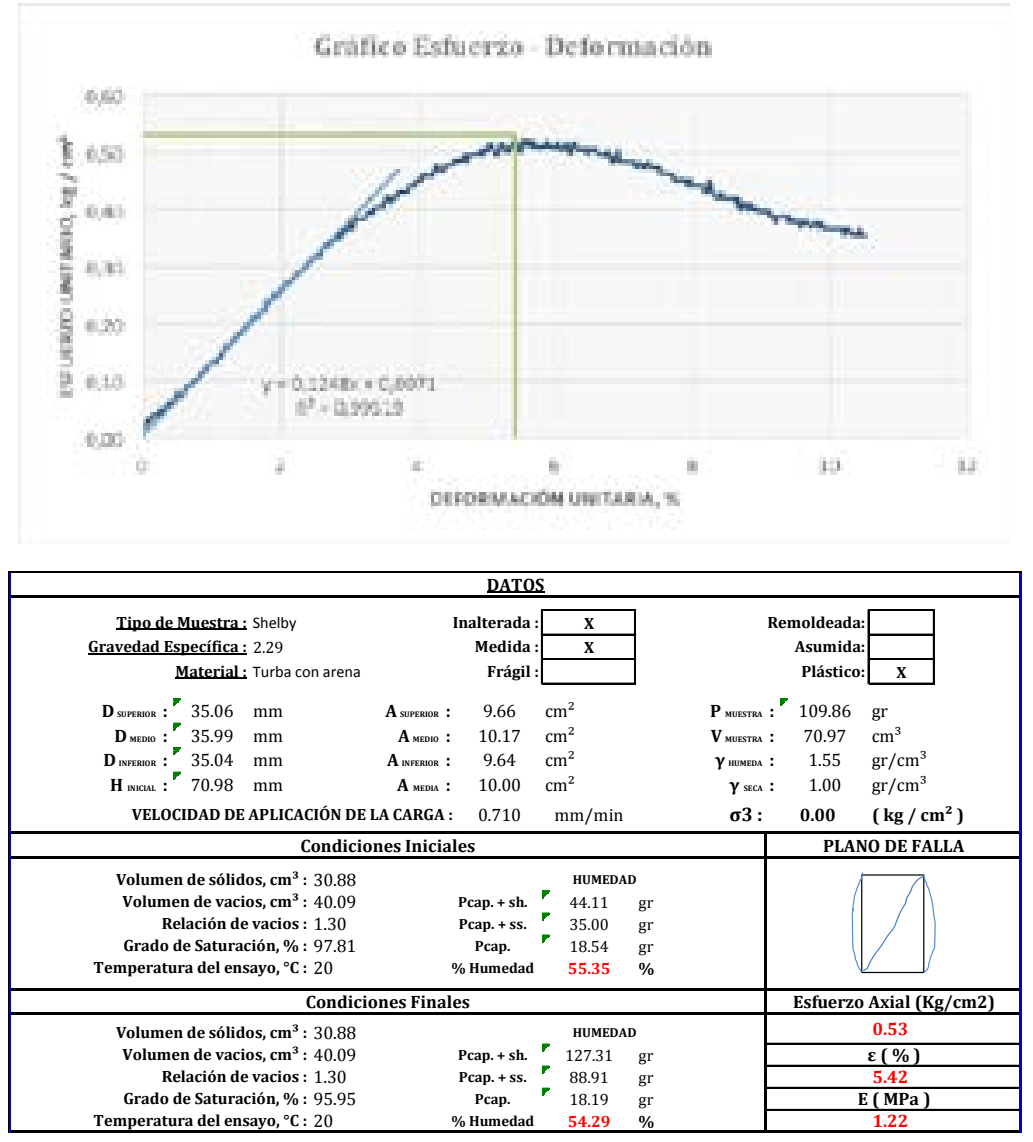


$C u_{L A B}=\frac{q u}{2}=\frac{0.53}{2}\left[\mathrm{~kg} / \mathrm{cm}^{2}\right]=0.265\left[\mathrm{~kg} / \mathrm{cm}^{2}\right]=C \boldsymbol{u}_{\boldsymbol{L A B}}=25.99[\mathrm{kPa}]$

- Muestra P1 de 2,50 a 3,00 m (2,90 a 3,00) de profundidad.

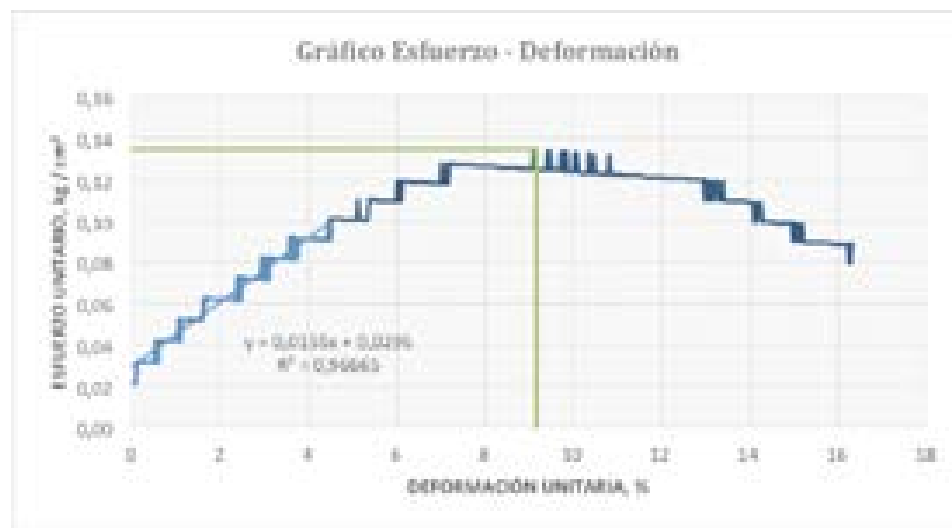

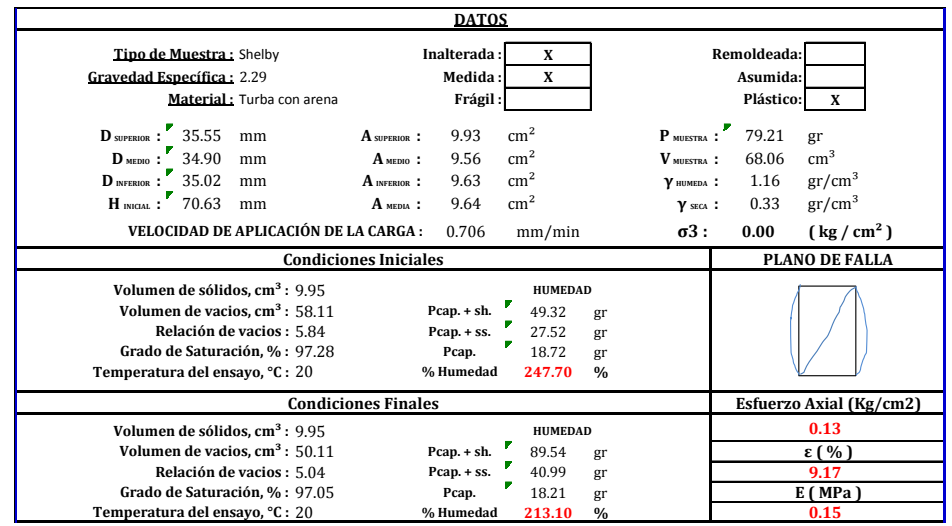

$$
C u_{L A B}=\frac{q u}{2}=\frac{0.13}{2}\left[\mathrm{~kg} / \mathrm{cm}^{2}\right]=0.065\left[\mathrm{~kg} / \mathrm{cm}^{2}\right]=\boldsymbol{C} \boldsymbol{u}_{\boldsymbol{L A B}}=\mathbf{6 . 3 7}[\mathrm{kPa}]
$$


Muestra P2 de 1,50 a 2,00 m (1,60 a 1,70) de profundidad.

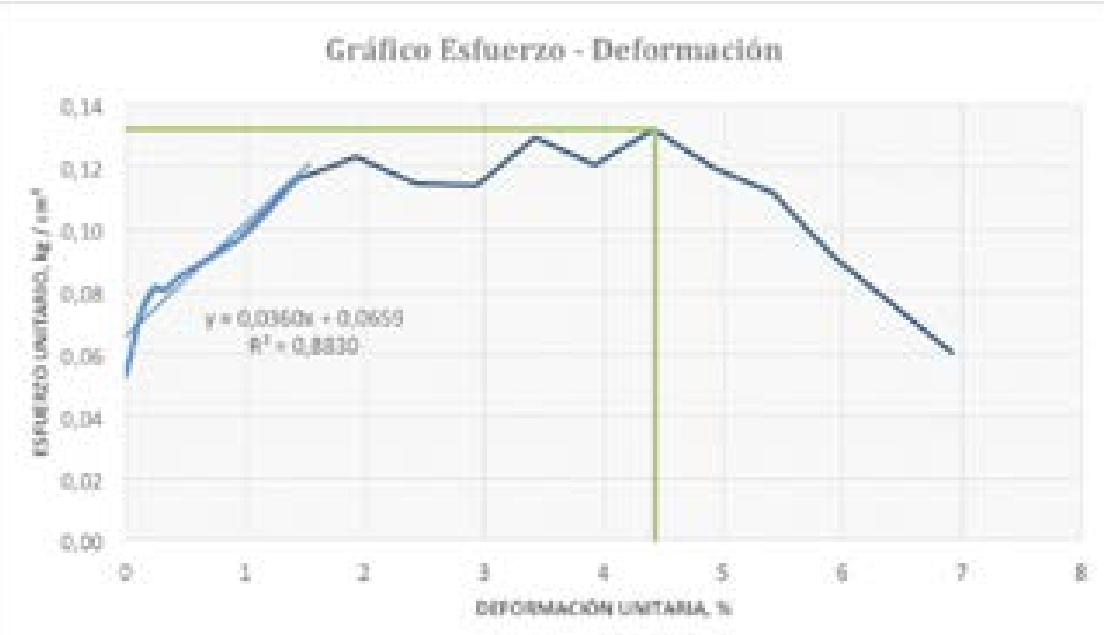

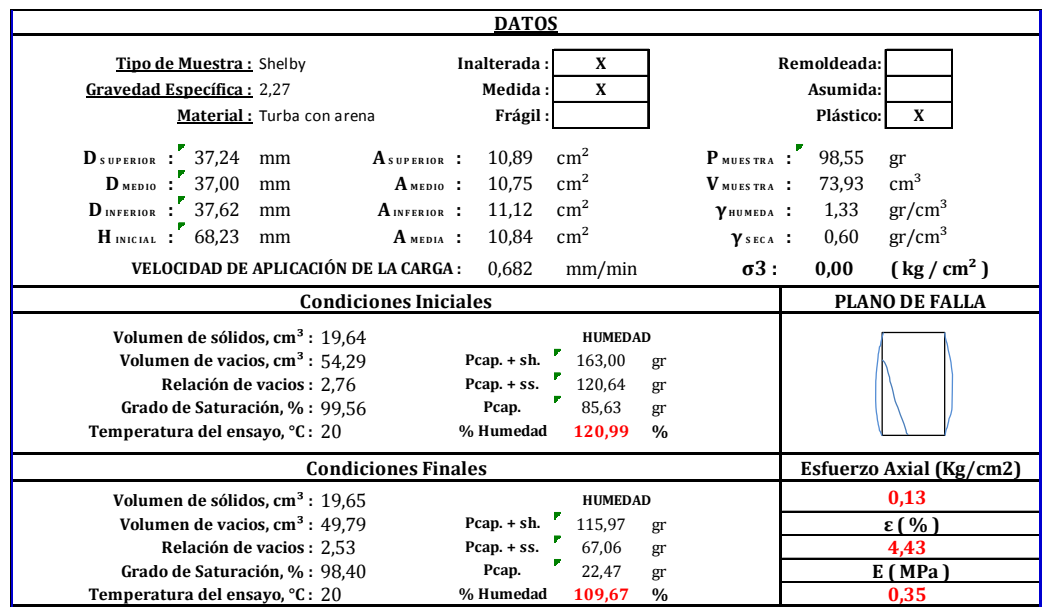

$$
C u_{L A B}=\frac{q u}{2}=\frac{0.13}{2}[\mathrm{~kg} / \mathrm{cm} 2]=0.065[\mathrm{~kg} / \mathrm{cm} 2]=C \boldsymbol{u}_{L A B}=\mathbf{6 . 3 7}[\mathrm{kPa}]
$$


Muestra P2 de 1,50 a 2,00 m (1,70 a 1,80) de profundidad.

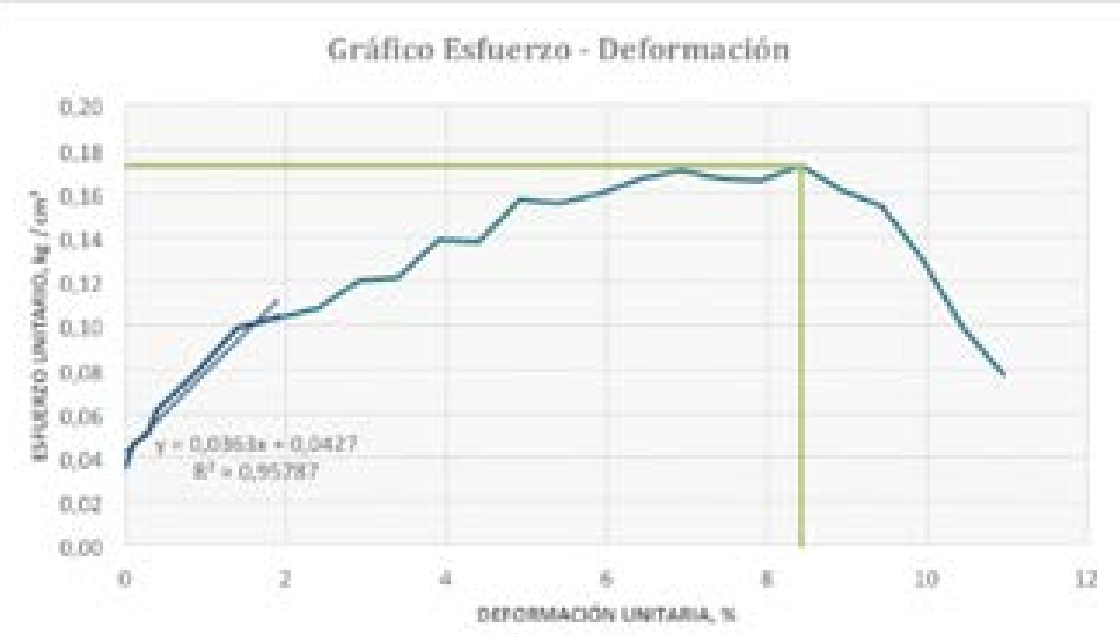

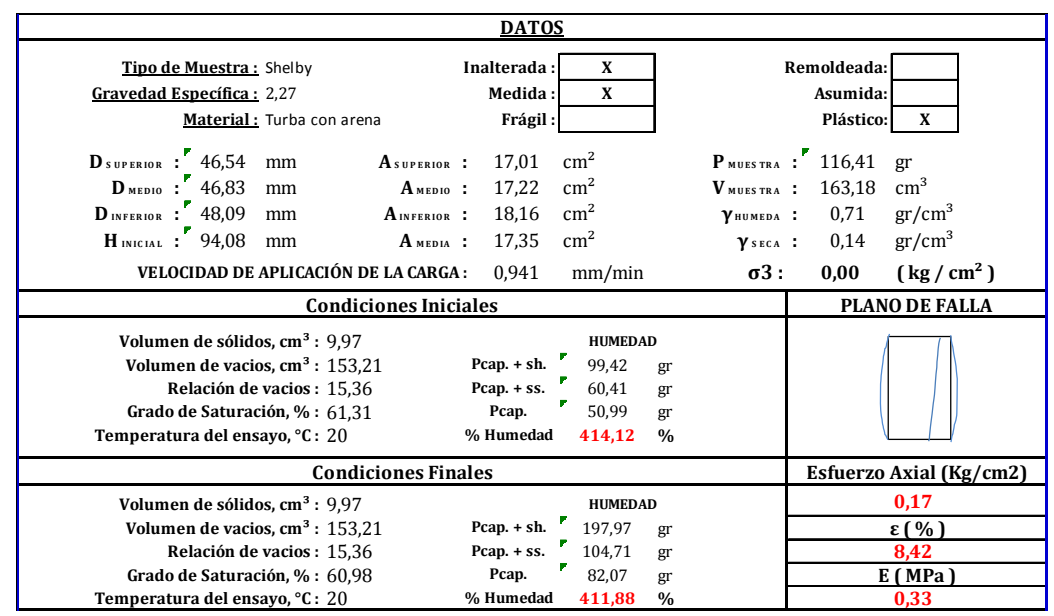

$$
C u_{L A B}=\frac{q u}{2}=\frac{0.17}{2}\left[\mathrm{~kg} / \mathrm{cm}^{2}\right]=0.085\left[\mathrm{~kg} / \mathrm{cm}^{2}\right]=\boldsymbol{C} \boldsymbol{u}_{\mathbf{L A B}}=\mathbf{8 . 3 4}[\mathrm{kPa}]
$$


Los resultados de laboratorio fueron comparados inicialmente con las correlaciones existentes entre el N SPT y Su (kPa) para suelos finos (Nassaji, 2011), obteniendo el gráfico que se presenta a continuación:

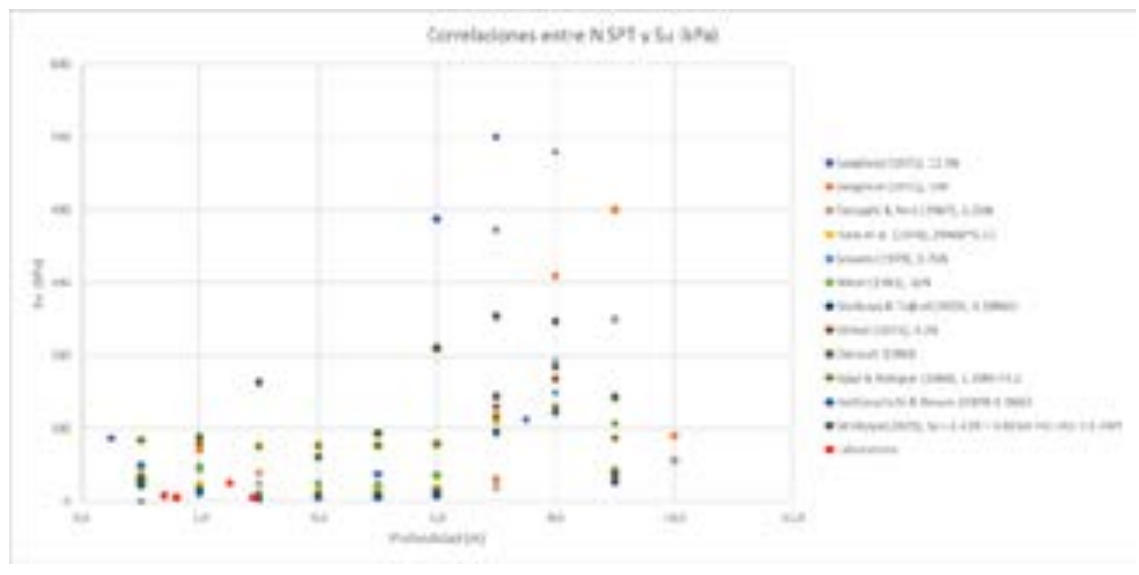

Figura n. ${ }^{0}$ 12. Correlación entre $N S P T$ y $S u(k P a)$.

Los valores tienen una alta dispersión, con rangos desde 4 a 500 kPa en los 7.5 metros prospectados. Con el fin de comparar con los resultados obtenidos en laboratorio, se modifica el eje Y para analizar los valores entre 0 y $50 \mathrm{kPa}$ : 


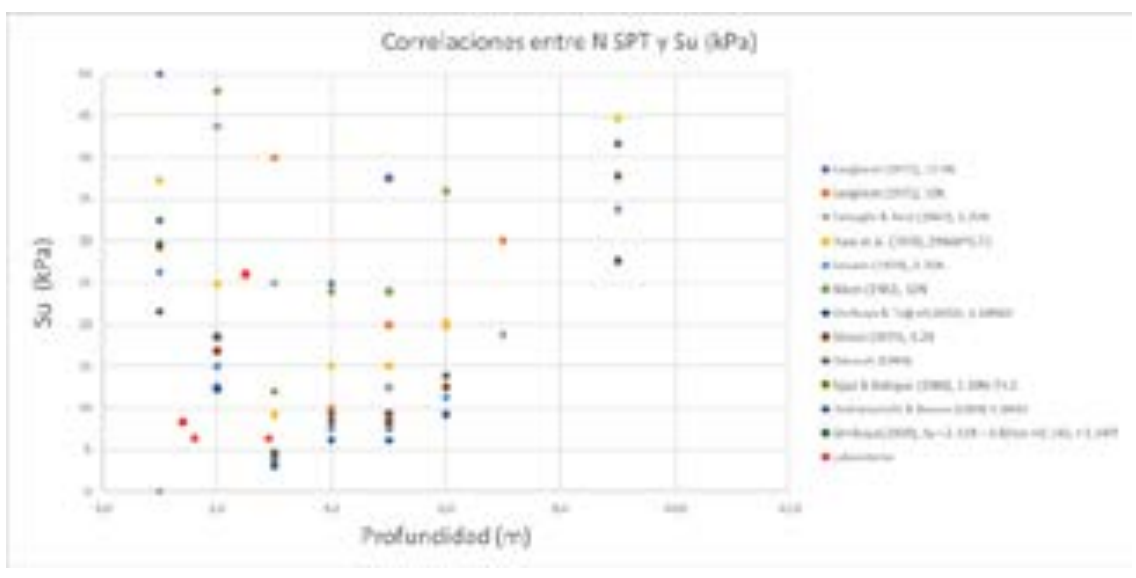

Figura n. ${ }^{\circ}$ 13. Correlación entre $N S P T$ y $S u(k P a)$. 


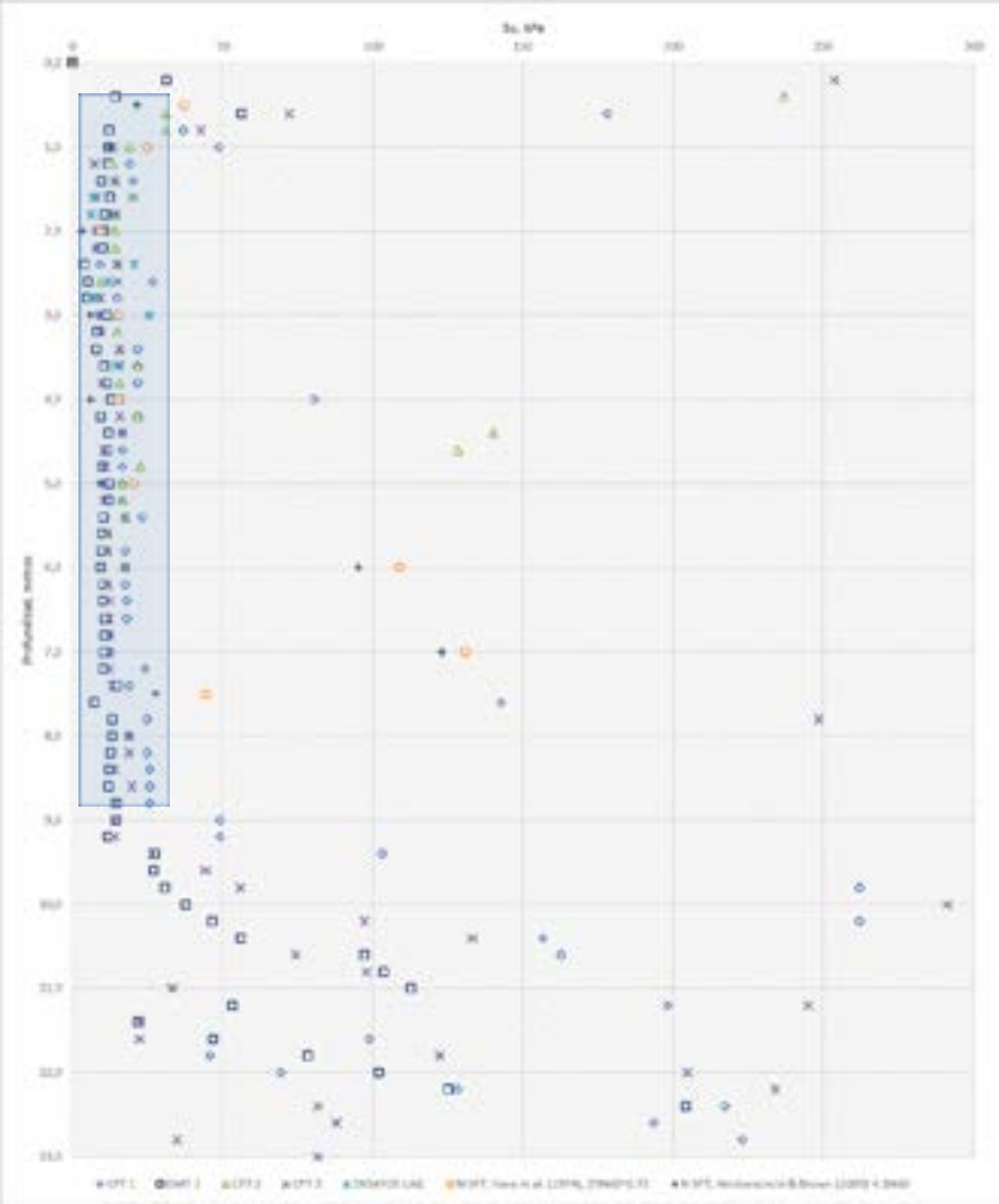

Figura n. ${ }^{\circ}$ 14. Interpretación de Cu para los diferentes métodos de ensayo aplicados. Rango de 0 a $200 \mathrm{kPa}$ 
En un análisis de los resultados presentados en un rango de 0 a $50 \mathrm{kPa}$ (Figura n.o 12), se puede observar que los resultados de 5 a $25 \mathrm{kPa}$ guardan una cercana aproximación, especialmente sobre el rango de 7 a $12 \mathrm{kPa}$, donde el Dilatómetro obtiene los resultados más conservadores de manera general, y la media de los valores del CPT dan como resultado los valores más cercanos a los obtenidos con ensayos de laboratorio. El análisis de estos resultados será presentado y discutido en el siguiente capítulo.

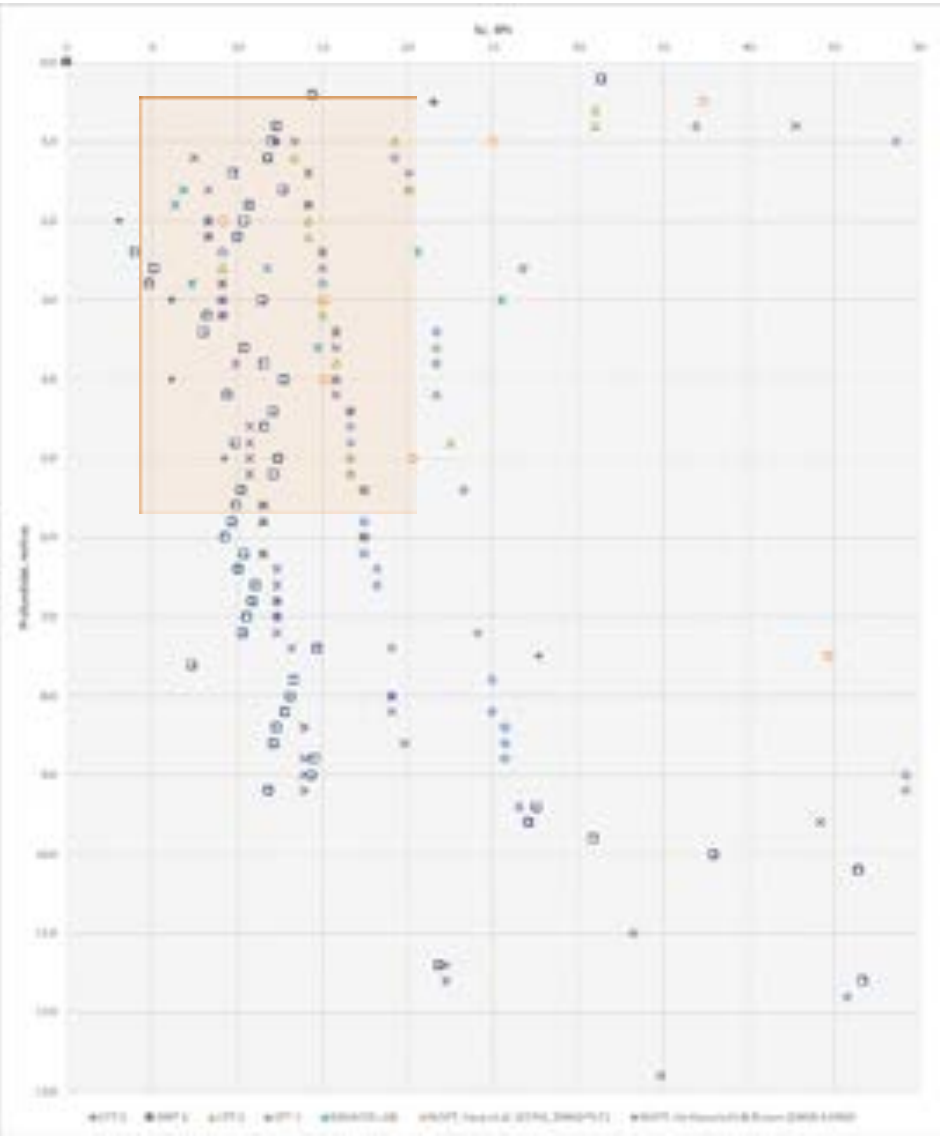

Figura n. ${ }^{\circ}$ 15. Interpretación de Cu para los diferentes métodos de ensayo aplicados. Rango de 0 a $50 \mathrm{kPa}$ 


\section{DISCUSIÓN Y CONCLUSIONES}

En la determinación del contenido orgánico y de humedad se observó una relación entre el incremento o disminución de ambos parámetros, lo cual confirma las investigaciones ya existentes donde se encontraron similares comportamientos (Haan \& Kruse, 2007). Sin embargo, en la profundidad prospectada se observó que la relación se ve afectada en el quinto y sexto metro, donde existen capas de arena intercaladas con los suelos orgánicos, lo cual puede servir de drenes y afectar la mencionada relación. La presencia de suelos minerales puede modificar cualquier posible correlación, y en investigaciones previas se ha encontrado que según el sitio, el contenido de humedad puede no ser de utilidad alguna para determinar el contenido orgánico o caracterizar a estos suelos (Nichol, 1998). Es importante mencionar que, debido a la fragilidad y estructura abierta de los suelos orgánicos y las turbas, el contenido de humedad medido en laboratorio puede que no sea el mismo que el contenido de humedad in situ (Davis, 1946).

Con las muestras alteradas obtenidas mediante el ensayo SPT se ejecutaron ensayos de límites de Atterberg, los cuales presentaron complejidad por la presencia de la propia materia orgánica. Los resultados obtenidos fueron erráticos, con una tendencia hacia un valor promedio entre 25 y $30 \%$ de contenido orgánico. Estos resultados fueron utilizados para aplicar la ecuación de Skempton y Petley (Skempton \& Petley, 1970), la cual correlaciona los resultados obtenidos entre el contenido orgánico y el límite de liquidez; sin embargo, no se observó similitud entre los resultados, al presentar una tendencia de incrementar los límites de liquidez y por ende contenido orgánico en función de la profundidad, con una pendiente promedio de 8.5 , por lo que se concluye que esta ecuación no es aplicable para los presentes suelos, es únicamente aplicable para los suelos donde fue estudiada. Investigaciones recientes (O'Kelly, 2015) concluyen que, a diferencia de los suelos minerales, los límites de Atterberg no son una fuente confiable para determinar la consistencia de suelos orgánicos, así como no tienen ninguna correlación aceptable con propiedades geomecánicas, lo cual se puede verificar con los datos erráticos obtenidos en esta investigación.

Los suelos orgánicos, al ser representados en los nomogramas del CPT (Robertson et al., 1986) y del DMT (Marchetti \& Crapps, 1981), se comportan como suelos minerales, clasificándose como arenas limosas, limos, limos arcillo- 
sos y arcillas en más de un $70 \%$, y para el $30 \%$ clasificado correctamente. Para poder ubicar los puntos, fue necesario modificar el nomograma de Robertson debido a los altos valores del coeficiente de Fricción (Figura 9). Similares resultados han sido obtenidos previamente (Zawrzykraj et al., 2017), donde se demostró que ninguno de los nomogramas presentados tanto para el DMT como para el CPT, sirven para la clasificación de suelos orgánicos de manera, y lo cual refleja la compleja naturaleza de estos suelos, tanto para su clasificación como para su caracterización mecánica.

La obtención de los valores de Cu en cada uno de los ensayos fue realizada a base de las ecuaciones disponibles en la bibliografía, donde se observa que, a lo largo de toda la profundidad, el DMT proporciona los valores más bajos, lo que se puede explicar porque la dilatación de la membrana es lateral, coincidiendo con la orientación del potencial de las fibras y eliminando cualquier posible refuerzo de las misma. Además, con el CPT se alcanzó la mayor cercanía a los valores de Cu obtenidos en laboratorio, lo cual puede deberse a la forma de ejecución del ensayo, donde se simula una compresión vertical, lo que permite que las fibras orientadas horizontales se tensen creando un refuerzo similar a un geotextil. Datos obtenidos mediante el DMT realizados en Polonia en turbas (Mlyna- rek, Wierzbicki, \& Bogucki, 2015), arrojen resultados casi idénticos al DMT probado en Ecuador, lo que puede deberse a factores de seguridad que Marchetti aplicó en su correlación (Marchetti \& Crapps, 1981).

Al considerar que en el Ecuador el principal ensayo para caracterización en sitio es el SPT, este fue ejecutado, aprovechando la obtención de las muestras alteradas para los otros ensayos. Correlaciones del N del SPT con Cu (o Su) son inexistentes para suelos orgánicos en la bibliografía, por lo que se aplicaron varias ecuaciones para suelos finos (Nassaji, 2011). Los resultados indican que no existe una correlación que se ajuste a todas las muestras de laboratorio, un ejemplo son los puntos a los 2.50 y a los 2.90 metros de profundidad, donde la resistencia al corte no drenada varía entre $26 \mathrm{kPa}$ a $6.37 \mathrm{kPa}$ respectivamente en 40 centímetros, variación que no es detectada por el N del SPT. De las gráficas se observa que no es posible obtener una correlación directa entre el N del SPT y el Su en suelos orgánicos, debido posiblemente a la variable descomposición, tipo y orientación de la materia orgánica, así como la heterogénea deposición de materiales en zonas con presencia de turbas y suelos orgánicos. Sin embargo, se obtienen resultados cercanos a la correlación propuesta por Hettiarachchi \& Brown (2009), la cual es presentada en 
las gráficas comparativas número 12 a 15. Debido a que no es el alcance de esta investigación, se recomienda que esta conclusión sea verificada con una mayor cantidad de muestras y datos en futuras investigaciones.

Al obtener valores cercanos de Cu entre los ensayos CPT y DMT, se graficó una correlación entre la resistencia del cono del CPT, $q_{c}$, con el módulo edométrico $M_{D M T}$, con base en la fórmula general que permite correlacionar ambas $M_{D M T}=a^{*} q_{c}$, para lo cual se obtuvo un valor de $a=8.5$. Similares correlaciones ya han sido ejecutadas, obteniendo valores $a=6.0$ para suelos orgánicos y turbas preconsolidadas con contenidos orgánicos de 50 al 92 \% (Zawrzykraj et al., 2017). La diferencia de contenido orgá- nico entre los suelos estudiados (cerca del $50 \%$ en promedio) podría explicar la diferencia de 2.5 en el valor de $a$, el cual se ha visto en investigaciones con arenas sobre consolidadas llega a valores de 8.41 (Balachowski, Kozak, \& Kurek, 2008). Se concluye que el valor de a propuesto debe ser estudiado más a fondo, y nuevas investigaciones con un mayor espectro de datos deben ser planteadas para verificar este valor.

El campo de investigación con métodos de ensayo como el Cono de Penetración Estático y el Dilatómetro de Marchetti en nuestro país aún no ha sido estudiado, por lo que sus ventajas y limitaciones en diversos tipos de suelos deben ser estudiadas para el beneficio de nuestra sociedad.

\section{AGRADECIMIENTOS}

El autor desea agradecer a la Pontificia Universidad Católica del Ecuador, y al Laboratorio de Materiales de Construcción de la Facultad de Ingeniería por financiar la Investigación "Uso del Cono de Penetración Estático en suelos finos", a partir de la cual se obtuvieron parte de los datos para esta investigación, así como a la Secretaría de Educación Superior, Ciencia, Tecnología e Innovación SENESCYT por financiar al autor para obtener el título de Master of Science in Soil Mechanics and Environmental Geotechnics en la Escuela Imperial de Londres. 


\section{BIBLIOGRAFÎA}

Ajlouni, M. A. (2000). Geotechnical properties of peat and related engineering problems. Ph.D. Thesis. Univ. of Illinois at Urbana- Champaign.

Alvarado, A. (1996). Evolución geológica cuaternaria y paleosismicidad de la Cuenca de Quito (Ecuador). Escuela Politécnica Nacional.

ASTM D1586-11. (2008). Standard Test Method for Standard Penetration Test (SPT) and Split-Barrel Sampling of Soils. ASTM Standard Test Method, D1586-08a, 1-9. https:// doi.org/10.1520/D1586-11.2

ASTM D3441 - 16. (2014). Standard Test Method for Mechanical Cone Penetration Tests of Soil. https://doi. org/10.1520/D3441

ASTM D6635 - 15. (2016). Standard Test Method for Performing the Flat Plate Dilatometer, 1-16. https:// doi.org/10.1520/D6635-15.2

Avilés, L. (2013). Caracterización Geológica - Geotectónica del sur de la ciudad de Quito, 190. Retrieved from http://www.dspace.uce. edu.ec/handle/25000/2752

Balachowski, L., Kozak, P., \& Kurek, N. (2008). Intercorrelation between CPTU-DMT tests for sands on the Baltic coast Intercorrelation between on the Baltic coast tests for, (September).
Boylan, Noel; Long, M. (Michael. (2012). In situ testing of peat - a review and update on recent developments Author ( $s$ ) version information. Geotechnical Engineering Journal of the SEAGS \& AGSSEA.

Burwash, A. L., \& Wiesner, W. R. (1984). CLASSIFICATION OF PEATS FOR GEOTECHNICAL ENGINEERING PURPOSES. (pp. 979-998). Retrieved from https://www. scopus.com/inward/record. uri?eid=2-s2.0-0021658317\&partnerID $=40 \& m d 5=a a 83703 d 0 a-$ b974103b62df48630fae80

Colleselli, F., Cortellazzo, G., \& Cola, S. (2000). Laboratory testing of Italian peaty soils. In ASTM Special Technical Publication (pp. 226-240). Retrieved from https:// www.scopus.com/inward/record. uri?eid=2-s2.0-0033903376\&partnerl $\mathrm{D}=40 \& \mathrm{md} 5=660$ e $3 \mathrm{~d}-$ 9588462dd703a18669efe1b451

Cuvi, N. (2017). Las ciudades como mosaicos bioculturales: el caso del Centro Histórico de Quito Las ciudades como mosaicos bioculturales : el caso del centro histórico de Quito, (May).

Davis, J. (1946). The peat deposits of Florida, their occurrence, development and uses ( FGS: Bulletin 
30). The Florida Geological Survey, $1-257$.

Dhowian, A. W., \& Edil, T. B. (1980). Consolidation Behavior of Peats. Geotechnical Testing Journal, 3(3), 105114. https://doi.org/10.1520/ GTJ10881J

Edil, T. B., Fox, P. J., \& Lan, L.-T. (1991). Endof-primary consolidation of peat. In Proceedings of the 10th European Conference on Soil Mechanics and Foundation Engineering, May 26, 1991 - May 30 (Vol. 1, pp. 65-68). Univ of Wisconsin-Madison, Madison, United States: Publ by A.A. Balkema.

Elsayed, A. (2006). Dilatometer \&amp; cone penetration tests on peat soil in Carver, Massachusetts. Civil Engineering Practice, 21(2).

Elsayed, A. A. (2003). No Title. The characteristics and engineering properties of peat in bogs. University of Massachusetts Lowell.

Haan, E. Den, \& Kruse, G. (2007). Characterisation and engineering properties of Dutch peats. ... of Characterisation and Engineering Properties of .... Retrieved from http://scholar. google.com/scholar?hl=en\&btn$\mathrm{G}=$ Search\&q=intitle:Characterisation+and+engineering+properties+of+Dutch+peats\#0

Hayashi, H., Nishimoto, S., \& Yamanashi, T. (2016). Applicability of settle- ment prediction method to peaty ground. Soils and Foundations. https://doi.org/10.1016/j.sandf.2016.01.012

Huat, B. B. K. (2014). Geotechnics of organic soils and peat. (A. Prasad author., A. Asadi author., \& S. Kazemian author., Eds.). Boca Raton: CRC Press.

Jarrett, P. M. (1983). Testing of Peats and Organic Soils. https://doi. org/10.1520/STP820-EB

Johari, N. N., Bakar, I., \& Aziz, M. H. A. (2015). Consolidation Parameters of Reconstituted Peat Soil: Oedometer Testing. Applied Mechanics and Materials, 773-774, 1466 1470. https://doi.org/10.4028/ wWw.scientific.net/AMM.773774.1466

Joint Nature Conservation Committee. (2011). Towards an assessment of the state of UK Peatlands. JNCC report.

Kamao, S. (2016). Creep and relaxation behavior of highly organic soil. International Journal of GEOMATE, 11(3), 2506-2511. Retrieved from https://www.scopus.com/inward/record.uri?ei$d=2-s 2.0-84958225892 \&$ partnerlD $=40 \& \mathrm{md} 5=5 \mathrm{a} 420044 \mathrm{bdd}-$ 107746f94cad381897293

Landva, A., \& La Rochelle, P. (1983). Compressibility and Shear Characte- 
ristics of Radforth Peats. Testing of Peats and Organic Soils, 157157-35. https://doi.org/10.1520/ STP37341S

Landva, A. O., Korpijaakko, E. O., \& Pheeney, P. E. (1983). GEOTECHNICAL CLASSIFICATION OF PEATS AND ORGANIC SOILS. In ASTM Special Technical Publication.

Landva, A. O., \& Pheeney, P. E. (1980). PEAT FABRIC AND STRUCTURE. Canadian Geotechnical Journal, 17(3), 416-435.

Ludeña, P. (2007). Variabilidad espacial del ensayo de penetración estandar en los sedimentos volcánicos del subsuelo del centro - norte de la ciudad de Quito.

Lunne, T., Robertson, P., \& Powell, J. (1997). Cone Penetration Testing in geotechnical practice.

Marchetti, S. (1980). In situ tests by flat dilatometer. Journal of the Geotechnical Engineering Division, ASCE, 106(GT3, Proc.).

Marchetti, S., \& Crapps, D. (1981). Flat Dilatometer Manual.

Mayne, P. W., Coop, M. R., Springman, S. M., Huang, A.-B., \& Zornberg, J. G. (2009). Geomaterial behavior and testing. In Proceedings of the 17th International Conference on Soil Mechanics and Geotechnical Engineering: The Academia and Practice of Geotechnical Engineering (Vol.
4). https://doi.org/10.3233/978-160750-031-5-2777

Mesri, G., \& Ajlouni, M. (2007). Engineering properties of fibrous peats. Journal of Geotechnical and Geoenvironmental Engineering, 133(7), 850-866. https:// doi.org/10.1061/(ASCE)10900241(2007)133:7(850)

Meyer, Z., Coufal, R., Kowalów, M., \& Szczygielski, T. (2011). Peat consolidation - new approach. Archives of Civil Engineering, LVII(2), 173. https://doi.org/http://dx.doi.org/10.2478/v.10169-011-0013-5

Mlynarek, Z., Wierzbicki, J., \& Bogucki, M. (2015). Geotechnical characterization of peat and gyttja by means of different in-situ tests. In 16th European Conference on Soil Mechanics and Geotechnical Engineering, ECSMGE 2015, September 13, 2015 - September 17 (Vol. 6, pp. 3097-3102). Pozna University of Life Sciences, Pozna, PolandAdam Mickiewicz University, Pozna, PolandILF Consulting Engineers, Poland: ICE Publishing.

Nassaji, F. (2011). SPT Capability to Estimate Undrained Shear Strength of Fine- Grained Soils of. Electronic Journal of Geotechnical Engineering, 16(January).

Nichol, D. (1998a). Construction Over Peat in Greater Vancouver, Bri- 
tish Columbia. Proceedings of the Institution of Civil Engineers - Municipal Engineer, 127(3), 109119. https://doi.org/10.1680/ imuen.1998.30986

Nichol, D. (1998b). Construction Over Peat in Greater Vancouver, British Columbia. Proceedings of the Institution of Civil Engineers - Municipal Engineer, 127(3), 109119. https://doi.org/10.1680/ imuen.1998.30986

O'Kelly, B. C. (2015). Atterberg limits are not appropriate for peat soils. Geotechnical Research, 2(3), 123134. https://doi.org/10.1680/jgere. 15.00007

Parish, F., Sirin, A., Charman, D., Joosten, $H_{\text {., }}$ Minayeva, T., Silvius, M., \& Stringer, L. (2008). Assessment on Peatlands, Biodiversity and Climate Change: Main Report. https://doi.org/10.1017/ CBO9781107415324.004

Peñafiel, L. (2008). Geología y Análisis del Recurso Hídrico subterráneo de la subcuenca del sur de Quito. Escuela Politécnica Nacional.

Radforth, N. W., \& Radforth, J. R. (1965). The significance of density as a physical property in peat deposits, 2(3), 81-88.

Rahgozar, M. A., \& Saberian, M. (2016). Geotechnical properties of peat soil stabilised with shredded was- te tyre chips. Mires and Peat, 18, 1-12. https://doi.org/10.19189/ MaP.2015.OMB.205 1

Robertson, P. K., Campanella, R. G., GiIlespie, D., \& Greig, J. (1986). USE OF PIEZOMETER CONE DATA.

Santander, P. (2013). Informe Técnico de Inspección Provincial Pichincha Quito Sector Turubamba.pdf.

Skempton, A. W., \& Petley, D. J. (1970). Ignition Loss and other Properties of Peats and Clays from Avonmouth, King's Lynn and Cranberry Moss. Géotechnique, 20(4), 343356. https://doi.org/10.1680/ geot.1970.20.4.343

Soper, E. K., \& Osbon, C. C. (1922). The occurrence and uses of peat in the United States. Bulletin. Retrieved from http://pubs.er.usgs.gov/publication/b728

Terzaghi, K., Peck, R. B., \& Mesri, G. (1996). Soil Mechanics in Engineering Practice.

Villagómez, D. (2014). Evolución Geológica Plio-cuaternaria del Valle Interandino Central en Ecuador (zona de Quito-Guayllabamba-San..., (May).

Wong, L. S. (2014). Unconfined compressive strength performance of cement stabilized peat with rice husk ash as a pozzolan. In Structural, Environmental (Vol. 567, pp. 545-550). Civil Engineering 
Department, College of Engineering, Jalan IKRAM-UNITEN, 43000 Kajang, Selangor, Malaysia: Trans Tech Publications Ltd. https://doi. org/10.4028/www.scientific.net/ AMM.567.545

Yamaguchi, H., Kawano, K., Teranishi, T., Matsui, K., \& Miura, Y. (1988). Shear properties of fibrous peat under three-dimensional stresses. In Proceedings of the International Conference on Engineering Problems of Regional Soils (p. 497). Beijing, China: Publ by Pergamon Press plc.

Yamaguchi, H., Ohira, Y., Kogure, K., \& Mori, S. (1985). Deformation and strength properties of peat. Proc. 11th international conference on soil mechanics and foundation engineering, San Francisco, August 1985. Vol. 4, (Balkema).

Yang, M., \& Liu, K. (2016). Deformation behaviors of peat with influence of organic matter. SpringerPlus, 5(1), 1-18. https://doi.org/10.1186/ s40064-016-2232-3

Zawrzykraj, P., Rydelek, P., \& Bąkowska, A. (2017). Geo-engineering properties of Eemian peats from Radzymin (central Poland) in the light of static cone penetration and dilatometer tests. Engineering Geology, 226, 290-300. https://doi.org/10.1016/j.enggeo.2017.07.001
Zhang, L., \& O'Kelly, B. C. (2014). The principle of effective stress and triaxial compression testing of peat. Proceedings of the Institution of Civil Engineers - Geotechnical Engineering, 167(1), 40-50. https://doi. org/10.1680/geng.12.00038

Zwanenburg, C., \& Jardine, R. J. J. (2015). Laboratory, in situ and full-scale load tests to assess flood embankment stability on peat. Géotechnique, 65(4), 309-326. https:// doi.org/10.1680/geot.14.P.257 
ill|lil 40

40 